Math. Model. Nat. Phenom.

Vol. 8, No. 5, 2013, pp. 131-154

DOI: $10.1051 / \mathrm{mmnp} / 20138509$

\title{
Instabilities and Dynamics of Weakly Subcritical Patterns
}

\author{
H.-C Kao*, E. Knobloch \\ Department of Physics, University of California, Berkeley CA 94720, USA
}

\begin{abstract}
The bifurcation to one-dimensional weakly subcritical periodic patterns is described by the cubic-quintic Ginzburg-Landau equation$$
A_{t}=\mu A+A_{x x}+i\left(a_{1}|A|^{2} A_{x}+a_{2} A^{2} A_{x}^{*}\right)+b|A|^{2} A-|A|^{4} A .
$$

These periodic patterns may in turn become unstable through one of two different mechanisms, an Eckhaus instability or an oscillatory instability. We study the dynamics near the instability threshold in each of these cases using the corresponding modulation equations and compare the results with those obtained from direct numerical simulation of the equation. We also study the stability properties and dynamical evolution of different types of fronts present in the protosnaking region of this equation. The results provide new predictions for the dynamical properties of generic systems in the weakly subcritical regime.
\end{abstract}

Keywords and phrases: bifurcation, cubic-quintic Ginzburg-Landau equation, weakly subcritical periodic pattern

Mathematics Subject Classification: 34E13, 37G40, 37M05

\section{Introduction}

The generic bifurcation of a homogeneous state to a stationary one-dimensional periodic pattern is described by the cubic real Ginzburg-Landau equation. This equation fails, however, in the vicinity of the transition from subcritical to supercritical behavior, i.e., when the cubic term responsible for the saturation of the instability is small. In this case the equation must be augmented by fifth order terms, and generically one finds that three additional terms enter into the description of the problem (see [1] and references therein). The resulting equation may be written in the form

$$
A_{t}=\mu A+A_{x x}+i\left(a_{1}|A|^{2} A_{x}+a_{2} A^{2} A_{x}^{*}\right)+b|A|^{2} A-|A|^{4} A .
$$

In the following we refer to this equation as the cubic-quintic Ginzburg-Landau equation or GL35 for short. This equation was first proposed in [2] and studied in [8,9]; its validity was analyzed rigorously in [3]. A generalization to two-dimensional systems with anisotropy, for example, for instabilities in a nematic liquid crystal, is given in [4].

\footnotetext{
${ }^{*}$ Corresponding author. E-mail: hckao@berkeley.edu
} 
Equation (1.1) describes the transition between subcritical and supercritical behavior in a number of physical systems of interest, including the Blasius boundary layer [2], binary fluid convection [6] and doubly diffusive convection [7], and can be used to predict the location of the snaking or pinning region in these systems within which spatially localized states are present [1]. Within Eq. (1.1) this region is associated with the presence of protosnaking behavior, i.e., the formation of a front between the trivial state $A=0$ and a large amplitude homogeneous pattern, corresponding to $|A| \neq 0$. In the following, we refer to the corresponding parameter value as a Maxwell point even though Eq. (1.1) is nonvariational whenever $a_{2} \neq 0$.

The paper is organized as follows. In section 2 we summarize the basic properties of Eq. (1.1). In section 3 we study the instabilities of periodic patterns, focusing on the subcritical regime. In particular, we derive evolution equations valid in the neighborhood of secondary instabilities of the primary wavetrain and compare their predictions with those obtained by direct numerical simulation of Eq. (1.1). Section 4 discusses the dynamics and stability of spatially localized solutions, including fronts, pulses and holes, near the Maxwell point. Brief conclusions follow in section 5. Appendix A summarizes the derivation of the cubic-quintic Ginzburg-Landau equation with $a_{2}=0$ from a generalized Swift-Hohenberg equation while Appendix B sketches the derivation of the nonlinear phase equation for the Eckhaus instability.

\section{Cubic-Quintic Ginzburg-Landau equation}

We are interested in the dynamics near the onset of a steady state pattern-forming instability in a translation- and reflection-invariant system with a base state $u=0$, and assume that this instability leads to a periodic state $u=u(x, t)$ with wavenumber $k=k_{c}$. Near the transition from supercritical to subcritical behavior $u(x, t)$ takes the form

$$
u(x, t)=\epsilon A\left(\epsilon^{2} x, \epsilon^{4} t\right) e^{i k_{c} x}+\text { c.c. }+ \text { h.o.t. }
$$

where $\epsilon \ll 1$ determines simultaneously the distance from the primary bifurcation and the degree of subcriticality of the primary bifurcation [1]. Under these conditions the amplitude $A$ satisfies Eq. (1.1). Here $\mu$ represents the bifurcation parameter, $b$ is a real $O(1)$ unfolding parameter, $a_{1}$ and $a_{2}$ are two real $O(1)$ coefficients, and the variables $x$ and $t$ now refer to $\epsilon^{2} x$ and $\epsilon^{4} t$. The translation invariance of the system is responsible for the equivariance of Eq. (1.1) with respect to the following operations

$$
\text { (i) } x \rightarrow x, A \rightarrow A e^{i \phi_{0}}, \quad \text { (ii) } x \rightarrow x+x_{0}, A \rightarrow A,
$$

while spatial reversibility is responsible for equivariance with respect to

$$
\text { (iii) } x \rightarrow-x, A \rightarrow A^{*} \text {. }
$$

Terms proportional to $i A_{x}$ may also be present but these can be eliminated by the change of variable $A \rightarrow A e^{-i \delta x}$ for a suitably chosen $\delta$. We remark that an equation of this type can be derived from the generalized Swift-Hohenberg equation near the primary pattern-forming instability [17,18]. A sketch of the derivation in the general case is provided in Appendix A.

As mentioned in the previous section, the term with coefficient $a_{2}$ renders the dynamics nonmonotonic. When $a_{2}=0$, Eq. (1.1) has gradient structure [1]

$$
A_{t}=-\frac{\delta F\left(A, A^{*}\right)}{\delta A^{*}}
$$

where the free energy $F$ takes the form

$$
F\left(A, A^{*}\right)=\int_{\Gamma}\left\{\left|A_{x}\right|^{2}-\mu|A|^{2}-\frac{b|A|^{4}}{2}+\frac{|A|^{6}}{3}+\frac{i a_{1}}{4}|A|^{2}\left(A A_{x}^{*}-A^{*} A_{x}\right)\right\} d x .
$$


The spatial domain $\Gamma$ can be either the real line or a finite interval with periodic boundary conditions (PBC). In the latter case the free energy is bounded from below if and only if $\left|a_{1}\right|<4 / \sqrt{3}[1]$ and in this case Eq. (1.1) is well posed. In the general case with nonzero $a_{2}$, global existence of smooth solutions has only been proved for $\left(a_{2}-a_{1}\right)^{2}<4[13]$.

Stationary solutions of Eq. (1.1) of the form $A(x)=R(x) e^{i \phi(x)}$ satisfy a completely integrable fourth order reversible dynamical system with two conserved quantities, $E$ and $L$,

$$
\begin{aligned}
& E \equiv R_{x}^{2}+U, \quad U(R ; \mu, L) \equiv \frac{L^{2}}{R^{2}}+\left(\mu+\frac{3 a_{2}-a_{1}}{2} L\right) R^{2}+\frac{b}{2} R^{4}+\beta R^{6}, \\
& L \equiv R^{2} \phi_{x}+\frac{a_{1}+a_{2}}{4} R^{4},
\end{aligned}
$$

where

$$
\beta \equiv \frac{\left(a_{1}+a_{2}\right)\left(3 a_{1}-5 a_{2}\right)}{48}-\frac{1}{3} .
$$

As a result, all stationary solutions can be found by examining the motions of a particle in the potential $U(R ; \mu, L)$. It follows that the only possible bounded stationary solutions are those corresponding to fixed points, periodic orbits, and homoclinic and heteroclinic connections, corresponding to $u(x)$ in the form of periodic wavetrains, quasiperiodic wavetrains, and localized states (pulses, holes and fronts), respectively.

\section{Stability and dynamics of periodic wavetrains}

Periodic wavetrains in $u$ correspond to fixed points in the spatial dynamics for $R$. These solutions have the form

$$
A_{0}(x)=R_{ \pm}\left(k_{0}\right) e^{i\left(k_{0} x+\phi_{0}\right)}, \quad R_{ \pm}^{2}\left(k_{0}\right) \equiv \frac{b^{\prime} \pm \sqrt{b^{\prime 2}+4\left(\mu-k_{0}^{2}\right)}}{2}
$$

where $k_{0}, \phi_{0}$ are constants and

$$
b^{\prime} \equiv b+k_{0}\left(a_{2}-a_{1}\right)
$$

and correspond to periodic wavetrains $u(x, t)$ with wavenumber $k_{c}+\epsilon^{2} k_{0}$. The bifurcation to such wavetrains is subcritical when $b^{\prime}$ is positive and supercritical when $b^{\prime}$ is negative. In the former case we refer to $R_{+}$as the upper branch, and $R_{-}$as the lower branch. Figure 1 shows the bifurcation diagram of periodic wavetrains in the subcritical region $\left(b^{\prime}>0\right)$. The linear stability of these states is studied in [1]; here we give a brief overview of the results.

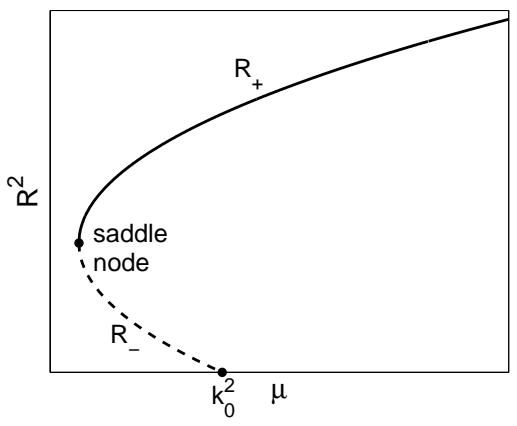

FiguRE 1. Bifurcation diagram showing $R^{2}$ as a function of $\mu$ in the subcritical region $\left(b^{\prime}>0\right)$. Solid (dashed) lines indicate solutions that are stable (unstable) in time with respect to amplitude perturbations. These correspond to $R_{+}$and $R_{-}$, respectively. 
We write

$$
A=A_{0}(1+a)
$$

with $a \equiv a(x, t)$ a small perturbation. This choice leads to the linearized equation

$$
\begin{aligned}
a_{t}= & a_{x x}+i\left(\frac{a_{1}-a_{2}}{2} R^{2}+\frac{2 L}{R^{2}}\right) a_{x}+i a_{2} R^{2} a_{x}^{*} \\
& +\left[b R^{2}+\left(\frac{a_{1}^{2}-a_{2}^{2}}{4}-2\right) R^{4}+\left(a_{2}-a_{1}\right) L\right]\left(a+a^{*}\right) .
\end{aligned}
$$

Since Eq. (3.4) does not depend explicitly on $x$ we may consider solutions of the form

$$
a=a_{q} \equiv \beta_{1} e^{\sigma t+i q x}+\beta_{2}^{*} e^{\sigma^{*} t-i q x},
$$

where $\beta_{1}$ and $\beta_{2}$ are complex numbers depending on $q$. The continuous eigenvalues $\sigma_{ \pm}(q)$ of the periodic wavetrain $u$ are given by

$$
\sigma_{ \pm}(q)=-\left(g+q^{2}\right) \pm \sqrt{\left(g+q^{2}\right)^{2}-q^{2}\left(f+q^{2}\right)},
$$

each of double multiplicity, where

$$
\begin{aligned}
& f\left(\mu, k_{0}\right) \equiv\left(4+a_{2}^{2}-a_{1}^{2}\right) R^{4}-\left(2 b^{\prime}+4 k_{0} a_{1}\right) R^{2}-4 k_{0}^{2}, \\
& g\left(\mu, k_{0}\right) \equiv 2\left(\mu-k_{0}^{2}\right)+b^{\prime} R^{2} .
\end{aligned}
$$

Thus when $q=0$ there is a marginal mode $a=i$ with eigenvalue $\sigma=0$ corresponding to translation invariance. The remaining mode is referred to as the amplitude mode and is stable when $g>0$ (i.e., on $R_{+}$) and unstable when $g<0$ (i.e., on $R_{-}$). The function $f$ can be computed directly from the potential $U$ and satisfies

$$
U_{R R}=-2 f
$$

on periodic wavetrains. This property plays an important role in characterizing the relationship between temporal and spatial stability when $q \neq 0$.

Let $w_{1}(q) \equiv q^{2} f+q^{4}$ and $w_{2}(q) \equiv g+q^{2}$. An eigenmode of wavenumber $q$ is stable if and only if both $w_{1}$ and $w_{2}$ are positive. This is so for all $q$ if and only if $f$ and $g$ are both nonnegative. This is so for periodic wavetrains $R=R_{+}$whenever $U_{R R}\left(R_{+}\right) \leq 0$. In this case localized states in the form of front, pulse and hole solutions connecting to $R_{+}$as $x \rightarrow \pm \infty$ may be stable since the background state to which they connect is itself stable.

Depending on $f$ and $g$, there are two qualitatively different ways for instabilities to occur:

(i) $f<0$ and $f \leq g$.

The curve $\left(w_{1}, w_{2}\right)(q)$ exits the stable region in the $\left(w_{1}, w_{2}\right)$ plane through $\left\{w_{1}=0, w_{2} \geq 0\right\}$. There is only one real unstable eigenvalue for each $q$ in the unstable region (figure $2(\mathrm{a})$ ).

(ii) $g<0$ and $f>g$.

The curve $\left(w_{1}, w_{2}\right)(q)$ exits the stable region through $\left\{w_{1} \geq 0, w_{2}=0\right\}$. If $f \geq 0$, there are always two unstable eigenvalues for each $q$ in the unstable region. The two unstable eigenvalues can be either a complex conjugate pair or two real eigenvalues (figure 2(b)). If $f<0$, there is only one unstable eigenvalue for each $q$ when $q^{2}<-f$ and two unstable eigenvalues when $q^{2}>-f$ for each $q$ in the unstable region.

In the following subsections we derive modulation equations describing the dynamics near the onset of each type of secondary instability and compare the resulting dynamics with results obtained via direct numerical simulation of Eq. (1.1). The time-stepping used here and in the subsequent sections employs the Fourier-based method ETD4RK [14]. Dealiasing is achieved via mode truncation. 

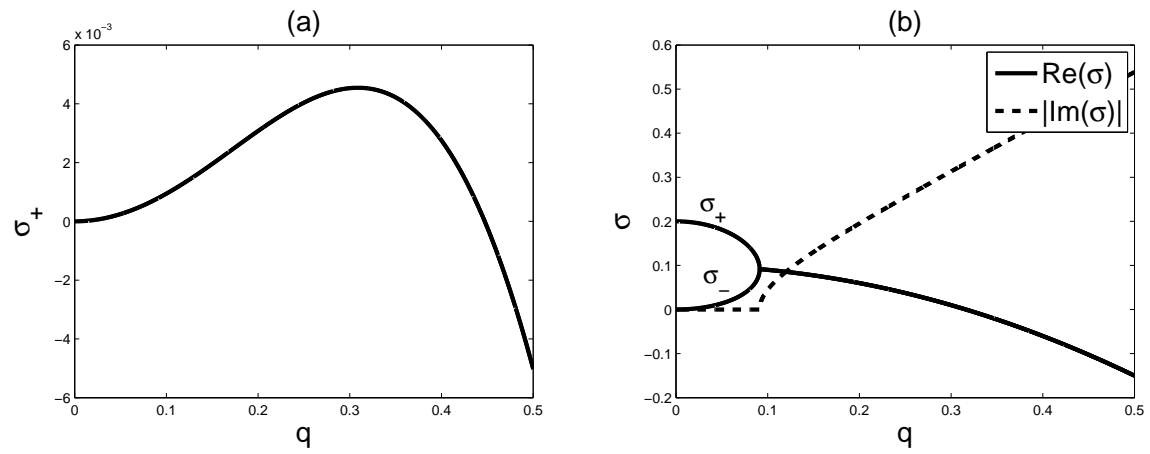

Figure 2. Eigenvalues $\sigma_{ \pm}$as functions of $q$ near the onset of secondary instability. (a) Phase mode: $f=-0.2$ and $g=1$. (b) Real and oscillatory modes: $f=1$ and $g=-0.1$.

\subsection{Phase mode: Eckhaus instability}

In this section we assume that the periodic state $R_{+}\left(k_{0}\right)$ is such that $g=O(1)>0$ and $f \approx 0$, i.e., $R_{+}\left(k_{0}\right)$ is close to the onset of instability. Figure 2(a) shows the eigenvalue $\sigma_{+}$versus the perturbation wavenumber $q$ when $f$ is slightly negative. The unstable modes are phase modes and result in Eckhaus instability, i.e., in phase slips [15].

To describe the initial stages of this instability we fix the coefficients $a_{1}, a_{2}$ and $b$, and treat $\mu$ as the primary bifurcation parameter. The instability is triggered by a change in the potential $U$ as $\mu$ or equivalently the amplitude $R_{+}\left(k_{0}\right)$ varies, and takes place when $f$ falls below $f=0$, or equivalently when $U_{R R}$ passes through zero, subject to the requirement that $k_{0}$, the asymptotic wavenumber at infinity, remains fixed. Thus $U=U(R ; \mu, L(\mu)) \equiv \tilde{U}(R ; \mu)$ and the instability sets in at $R_{+}\left(k_{0}\right)=R_{0,+}$, where

$$
R_{0, \pm}^{2}=\frac{b^{\prime}+2 k_{0} a_{1} \pm \sqrt{\left(b^{\prime}+2 k_{0} a_{1}\right)^{2}+4\left(4+a_{2}^{2}-a_{1}^{2}\right) k_{0}^{2}}}{4+a_{2}^{2}-a_{1}^{2}}>0, \quad \mu_{0}=R_{0}^{4}-b^{\prime} R_{0}^{2}+k_{0}^{2} .
$$

The branching direction of the stationary modulated states that result can be readily determined by a local expansion of $\tilde{U}$ around $\left(R_{0}, \mu_{0}\right)$. We write $(R, \mu)=\left(R_{0}+\delta R, \mu_{0}+\delta \mu\right)$ and suppose that generically $\tilde{U}_{R R R, 0} \tilde{U}_{R \mu, 0} \neq 0$. Thus

$$
\tilde{U}(R ; \mu)=\tilde{U}\left(R_{0} ; \mu\right)+\tilde{U}_{R \mu, 0} \delta \mu \delta R+\frac{\tilde{U}_{R R R, 0}}{6}(\delta R)^{3}+\text { h.o.t. }
$$

where $\delta R=O\left(|\delta \mu|^{1 / 2}\right)$ and the subscript 0 indicates that the quantity is evaluated at the bifurcation point. The bifurcation occurs as $\mu$ increases (decreases) provided $\tilde{U}_{R R R, 0} \tilde{U}_{R \mu, 0}$ is negative (positive); the corresponding stationary solution is given by

$$
\delta R \equiv \pm\left(-\frac{2 \tilde{U}_{R \mu, 0} \delta \mu}{\tilde{U}_{R R R, 0}}\right)^{1 / 2}\left[3 \operatorname{sech}^{2}(h x)-1\right]+O(|\delta \mu|), \quad h \equiv \frac{1}{2}\left(-\frac{1}{2} \tilde{U}_{R R R, 0} \tilde{U}_{R \mu, 0} \delta \mu\right)^{1 / 4}
$$

where the positive sign corresponds to a pulse or a hole solution according to whether $\tilde{U}_{R R R, 0}>0$ or $\tilde{U}_{R R R, 0}<0$ (see Eq. (3.10)). In the present case Eq. (2.4) together with the fact that $\tilde{U}_{R R, 0}=0$ at the bifurcation point imply that

$$
\tilde{U}_{R \mu, 0}=2 R_{0}, \quad \tilde{U}_{R R R, 0}=24 R_{0}\left(b+8 \beta R_{0}^{2}\right) .
$$

Thus $\tilde{U}_{R \mu, 0}$ is always positive and the bifurcated solution is a hole with branching to the right (or pulse with branching to the left) whenever $b+8 \beta R_{0}^{2}$ is negative (or positive). 
When the periodic wavetrain lies at the band center, i.e., $k_{0}=0$, the bifurcation is at

$$
\left(R_{0}^{2}, \mu_{0}\right)=\left(\frac{2 b}{4+a_{2}^{2}-a_{1}^{2}}, \frac{2 b^{2}\left(a_{1}^{2}-a_{2}^{2}-2\right)}{\left(4+a_{2}^{2}-a_{1}^{2}\right)^{2}}\right)
$$

and hence lies close to the saddle-node $\left(R^{2}, \mu\right)=\left(b / 2,-b^{2} / 4\right)$ whenever $\left|a_{1}^{2}-a_{2}^{2}\right| \ll 1, b>0$. Since $\tilde{U}_{R R R, 0}$ is now equal to $-8 R_{0}^{3}\left[2+a_{2}\left(a_{1}+a_{2}\right)\right]$ it follows that the bifurcating solution is a hole branching to the right when $2+a_{2}\left(a_{1}+a_{2}\right)>0$, or a pulse branching to the left when $2+a_{2}\left(a_{1}+a_{2}\right)<0$.

To describe the dynamics that result we use multiple scale analysis to derive an evolution equation for the phase of the pattern. When $\partial_{\mu} f\left(\mu_{0}, k_{0}\right) \neq 0, f$ can be approximated as

$$
f=\left.\partial_{\mu} f\right|_{\mu=\mu_{0}} \delta \mu+o(|\delta \mu|)=\frac{\left[\left(4+a_{2}^{2}-a_{1}^{2}\right) R_{0,+}^{2}+4 k_{0}^{2} / R_{0,+}^{2}\right]}{\sqrt{b^{\prime 2}+4\left(\mu_{0}-k_{0}^{2}\right)}} \delta \mu+o(|\delta \mu|),
$$

where $R_{0,+}$ is $R_{+}$evaluated at $(\mu, k)=\left(\mu_{0}, k_{0}\right)$. If $a_{1}^{2}-a_{2}^{2}<4,\left.\partial_{\mu} f\right|_{\mu=\mu_{0}}$ is always positive implying that $R_{0,+}$ is Eckhaus-stable for $\mu>\mu_{0}$. In contrast, if $a_{1}^{2}-a_{2}^{2}>4$, the quantity $\left.\partial_{\mu} f\right|_{\mu=\mu_{0}}$ can be negative in an appropriate region of the $\left(a_{1}, a_{2}\right)$ plane and this allows the phase mode to be unstable when $\mu>\mu_{0}$. There is no instability when $\mu<\mu_{0}$.

In the nondegenerate case $\partial_{\mu} f\left(\mu_{0}, k_{0}\right)=O(1)$ we assume that $\delta \mu=O\left(\epsilon^{2}\right)$ and require that the $q^{2}$ term in Eq. (3.5) is comparable with $f$, i.e., $q=O(\epsilon)$. The corresponding timescale is determined by the magnitude of the most unstable eigenvalue which is $O\left(\epsilon^{4}\right)$. For eigenmodes near $q=0$, the real part of the eigenvector is $O(\epsilon)$ smaller than its imaginary part implying that the instability results primarily in phase modulation. We therefore write

$$
\begin{aligned}
& \delta \mu=\epsilon^{2} \mu_{2}, \quad T=\epsilon^{4} t, \quad X=\epsilon x, \\
& A=R_{+}\left(1+\epsilon^{2} r^{(0)}+\epsilon^{4} r^{(1)}+\ldots\right) e^{i\left(k_{0} x+\epsilon \phi^{(0)}+\epsilon^{3} \phi^{(1)}+\ldots\right),}
\end{aligned}
$$

where $r^{(n)}$ and $\phi^{(n)}$ are real functions of $X$ and $T$. It can now be shown that with this scaling the phase perturbation $\phi^{(0)}$ satisfies the nonlinear phase equation [12]

$$
2 g_{0} \phi_{T}^{(0)}=-\phi_{X X X X}^{(0)}+f_{2} \phi_{X X}^{(0)}+2 \gamma \phi_{X}^{(0)} \phi_{X X}^{(0)},
$$

where $f_{2} \equiv f / \epsilon^{2}=O(1),\left.g_{0} \equiv g\right|_{\mu_{0}, k_{0}}=O(1)$ and

$$
\gamma=\frac{\tilde{U}_{R R R, 0} R_{0}}{4 b_{+}}, \quad b_{ \pm} \equiv a_{2} R_{0}^{2} \pm\left(a_{1} R_{0}^{2}+2 k_{0}\right) .
$$

The perturbed amplitude $r^{(0)}$ is slaved to $\phi_{X}^{(0)}$,

$$
2 g_{0} r^{(0)}=b_{-} \phi_{X}^{(0)} .
$$

These results can also be written in terms of the perturbation wavenumber $\kappa \equiv \phi_{X}^{(0)}$,

$$
2 g_{0} \kappa_{T}=\left(-\kappa_{X X}+f_{2} \kappa+\gamma \kappa^{2}\right)_{X X} .
$$

A sketch of the derivation is given in Appendix B.

Equation (3.19) has stationary spatially localized solutions in the Eckhaus-stable regime $f_{2}>0$. In the stationary case, the phase equation can be integrated twice, yielding

$$
-\kappa_{X X}+f_{2} \kappa+\gamma \kappa^{2}=0 .
$$


The integration constants are set to zero by the requirement that the asymptotic wavenumber outside the structure remains unchanged. Bifurcation to localized solutions takes place as $f_{2}$ becomes positive and the resulting solution takes the form

$$
\kappa_{0}(X)=-\frac{3 f_{2}}{2 \gamma} \operatorname{sech}^{2}\left(\frac{\sqrt{f_{2}}}{2} X\right) .
$$

Within the phase description the stability properties of this solution follow from the fact that Eq. (3.19) has a Lyapunov functional,

$$
G[\kappa]=\int_{\mathbb{R}}\left(\frac{\kappa_{X}^{2}}{2}+\frac{f_{2}}{2} \kappa^{2}+\frac{\gamma}{3} \kappa^{3}\right) d X
$$

i.e., $G[\kappa]$ decreases monotonically with time. Consider $\tilde{G}(s)=G\left[\kappa_{0}+s w\right]$, where $w$ is an arbitrary smooth function that tends to 0 as $|X| \rightarrow \infty$. Locally around $s=0$, we have

$$
\tilde{G}(s)=\tilde{G}(0)+\frac{1}{2} \tilde{G}^{\prime \prime}(0) s^{2}+O\left(s^{3}\right),
$$

where $\tilde{G}^{\prime \prime}(0)$ takes the form

$$
\tilde{G}^{\prime \prime}(0)=\frac{\sqrt{f_{2}}}{2} \int_{\mathbb{R}}\left\{\frac{w_{X^{\prime}}^{2}}{2}+\left(2-6 \operatorname{sech}^{2} X^{\prime}\right) w^{2}\right\} d X^{\prime}, \quad X^{\prime}=\frac{\sqrt{f_{2}}}{2} X .
$$

Consider now the eigenvalue problem

$$
-w^{\prime \prime}+\left(4-12 \operatorname{sech}^{2} X^{\prime}\right) w=\lambda w .
$$

It is known [19] that Eq. (3.25) has one negative eigenvalue, $\lambda=-5$. It follows, therefore, that $\tilde{G}^{\prime \prime}(0)<0$ for a suitable choice of $w$ and hence that the solution given in Eq. (3.21) is unstable. The presence of an unstable localized state for $f>0$ is consistent with the well-known fact that the Eckhaus instability is subcritical.

We now turn to the study of the dynamical evolution of the Eckhaus instability that takes place when $f<0$ using direct numerical integration of Eq. (1.1) for different choices of $\left(a_{1}, a_{2}\right)$ with $a_{1}-a_{2}, k_{0}, g>0$ and $f<0$ fixed. The reason for fixing $f$ and $g$ is to ensure that the spectrum of the linearized equation remains unchanged for different choices of $\left(a_{1}, a_{2}\right)$ and this can be done by modifying the values $\mu$ and $b$. We pick $a_{1}-a_{2}=0.45, f=-0.02, g=1.3, k_{0}=\pi / 10$ with domain size $\Gamma=200$ and periodic boundary conditions. The number of grid points, here and elsewhere, is 512, with time step equal to 0.01. We examine the cases $a_{1}=0.65,0.75,1.25$, and 2.25 with $\gamma \approx-7.3351,-6.1736,-4.3349$, and -3.9596 . The initial condition we choose is a periodic wavetrain with a superimposed perturbation $a=c_{1} e^{i q x}+c_{2} e^{-i q x}$, $q=6 \pi / \Gamma$, corresponding to the most unstable mode in the given domain. Time evolution of the profile of $\operatorname{Re}[A]$ is shown in figure 3 initialized with perturbation amplitude $\sqrt{c_{1}^{2}+c_{2}^{2}}=0.05$. With the given initial condition, the number of rolls changes from 10 to 7 as a result of phase slips triggered by the Eckhaus instability, and it is clear that the time for a phase slip to occur is smaller when $|\gamma|$ is larger. Phase slips occur at $t=T \approx 1860,2465,4380$ and 5075, respectively; for the perturbation amplitude $\sqrt{c_{1}^{2}+c_{2}^{2}}=0.05$ the time $T$ is determined largely by nonlinear processes and not by the linear growth phase of the instability.

In figure 4 we examine the dependence of these results on initial conditions. The figure shows the dynamics of an Eckhaus-unstable 10-roll periodic wavetrain with a superposed unstable mode with perturbation wavenumber (a) $2 \pi / \Gamma$, (b) $4 \pi / \Gamma$, (c) $6 \pi / \Gamma$, and (d) $8 \pi / \Gamma$. The simulation shows that the number of rolls decreases by $1,2,3$ and 4 . Here $a_{1}=0.75, a_{2}=0.3$ and the remaining quantities are as in figure 3.

We also investigate the dependence of the time to phase slip on the linear growth rate $\sigma_{+}(q)$. Here we fix $a_{2}=0.2, g=1.3, \gamma=-7$, and choose the wavenumber of the periodic wavetrain $k_{0}=\pi / 10$. Values 


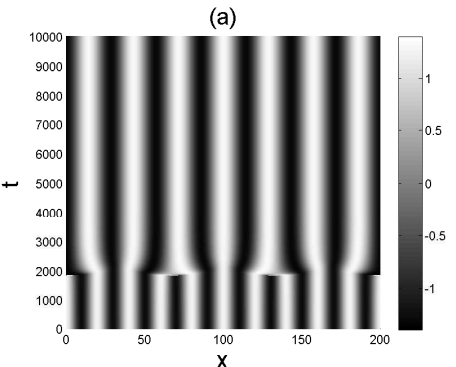

(c)

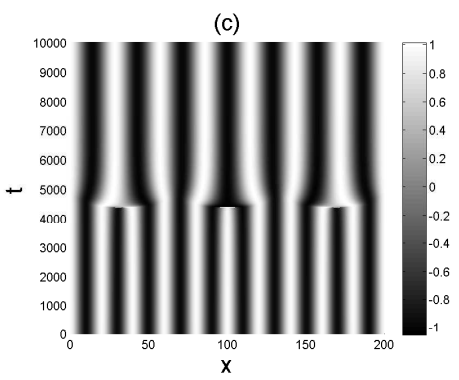

(b)

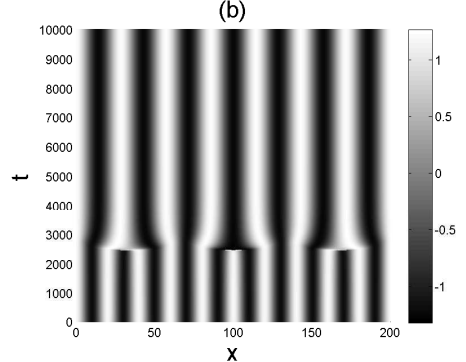

(d)

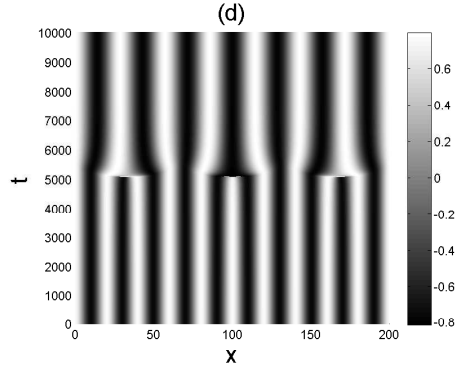

Figure 3 . Time evolution of $\operatorname{Re}[A]$ with $\mathrm{PBC}$ in a domain of size $\Gamma=200$ triggered by the Eckhaus instability. Parameters: $f=-0.02, g=1.3, k_{0}=\pi / 10$, and $a_{1}-a_{2}=0.45$. (a) $a_{1}=0.65$. (b) $a_{1}=0.75$. (c) $a_{1}=1.25$. (d) $a_{1}=2.25$. In each case the solution has been initialized with a sinusoidal perturbation of amplitude $\sqrt{c_{1}^{2}+c_{2}^{2}}=0.05$ and wavenumber $q=6 \pi / \Gamma$.

of the parameters $a_{1}, \mu$, and $b$ are adjusted so that the numerical value of $f$ is approximately equal to $-0.01503,-0.01751,-0.01999$, and -0.02247 . In each case the most unstable mode corresponds to $q=6 \pi / \Gamma$ with the corresponding temporal eigenvalue $\sigma_{+} \approx 2.0847 \times 10^{-5}, 2.9261 \times 10^{-5}, 3.7680 \times 10^{-5}$, and $4.6105 \times 10^{-5}$, respectively. Figure 5 shows the time evolution resulting from an initial perturbation with $\sqrt{c_{1}^{2}+c_{2}^{2}}=0.001$; figure 6 confirms that for these small amplitudes the time $T$ to phase slip is determined by the linear growth rate $\sigma_{+}^{-1}$. This is no longer the case for perturbations with amplitude $\sqrt{c_{1}^{2}+c_{2}^{2}} \gtrsim 0.01$, cf., figure 4 .

The degenerate situation $f\left(\mu_{0}, k_{0}\right)=\partial_{\mu} f\left(\mu_{0}, k_{0}\right)=0$ (Eq. (3.6)) arises when $a_{1}^{2}-a_{2}^{2}>4$ and is always present in the supercritical case $\left(b^{\prime}<0\right)$. In the subcritical case $\left(b^{\prime}>0\right)\left|a_{1}\right|$ must be smaller than $\left|a_{2}\right|+2 /\left|a_{2}\right|$ for this degeneracy to occur. At this degenerate point $R_{0,+}$ and $b$ are given by

$$
R_{0,+}^{2}=2\left|k_{0}\right|\left(a_{1}^{2}-a_{2}^{2}-4\right)^{-1 / 2}, \quad b=-2\left|k_{0}\right|\left(a_{1}^{2}-a_{2}^{2}-4\right)^{1 / 2}-k_{0}\left(a_{1}+a_{2}\right) .
$$

It can be shown that at this point $\partial_{\mu}^{2} f\left(\mu_{0}\right)$ is always negative implying that the periodic state is always unstable when $\mu$ is slightly perturbed from $\mu_{0}$. The derivation of the phase equation corresponding to this case is similar to the nondegenerate case except that $\delta \mu=O(\epsilon)$ instead of $O\left(\epsilon^{2}\right)$, and also results in Eq. (3.16).

The degeneracy $\gamma=0$ (Eqs. (3.12) and (3.17)) is of greater interest. This degeneracy only arises when $\beta>0$ (Eq. (2.6)) implying that $\left(a_{1}, a_{2}\right)$ must lie in the range $\left|a_{1}-a_{2}\right|>2$. We remark that Eq. (1.1) may not be well-posed in this regime. From Eq. (3.19), we can see that in order for the next higher order term $\left(\kappa^{3}\right)_{X X}$ to come in, the scaling must be modified:

$$
X=\epsilon x, \quad T=\epsilon^{4} t, \quad \kappa=O(\epsilon), \quad \gamma=\epsilon \gamma_{1} .
$$

The size of $\gamma$ is controlled by varying $b$. Since $\kappa$ is $O(\epsilon)$, the perturbations of the phase and amplitude will be $O(1)$ and $O(\epsilon)$, respectively, so that

$$
A=R_{+}\left(1+\epsilon r^{(0)}+\epsilon^{3} r^{(1)}+\ldots\right) e^{i\left(k_{0} x+\phi^{(0)}+\epsilon^{2} \phi^{(1)}+\ldots\right)} .
$$



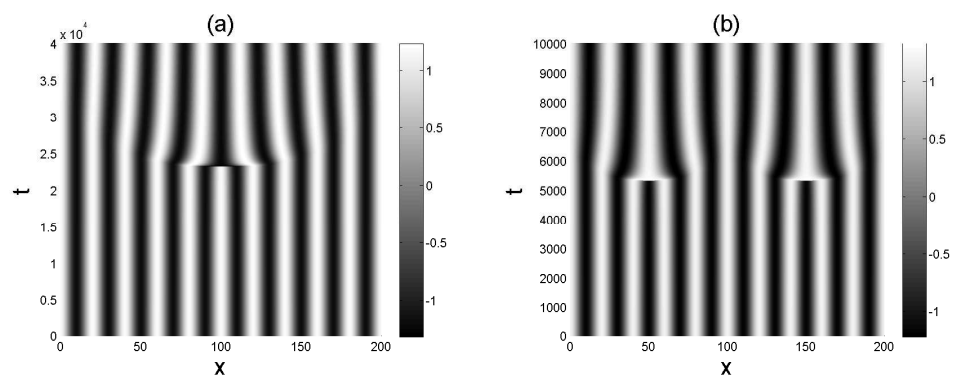

(c)
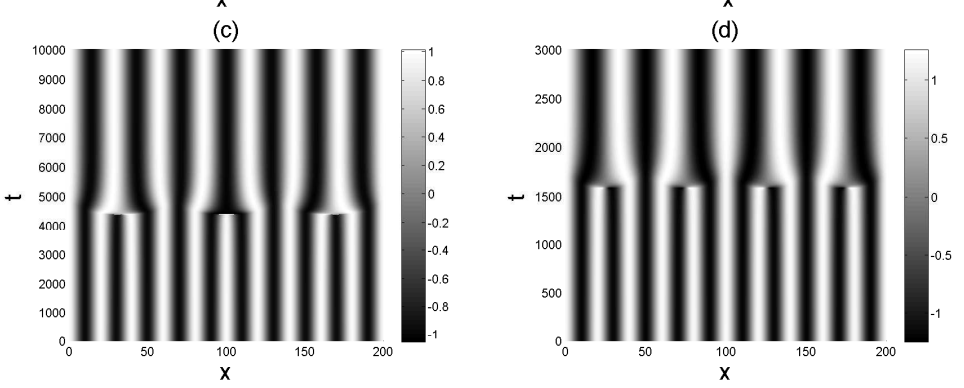

Figure 4 . Time evolution of $\operatorname{Re}[A]$ with $\mathrm{PBC}$ in a domain of size $\Gamma=200$ triggered by the Eckhaus instability. Parameter values are $a_{1}=0.75, a_{2}=0.3$, with the remaining quantities as in figure 3 . The superposed initial perturbation is periodic with wavenumber (a) $2 \pi / \Gamma$, (b) $4 \pi / \Gamma$, (c) $6 \pi / \Gamma$, (d) $8 \pi / \Gamma$, and amplitude $\sqrt{c_{1}^{2}+c_{2}^{2}}=0.05$ in each case.

Following the procedure in the nondegenerate case, one can show that the perturbed wavenumber $\kappa$ satisfies the Cahn-Hilliard equation but with a negative cubic coefficient,

$$
2 g_{0} \kappa_{T}=\left[-\kappa_{X X}+f_{2} \kappa+\gamma_{1} \kappa^{2}-2\left(1+2 k_{0} / b_{+}\right)^{2} \kappa^{3}\right]_{X X},
$$

cf., Eq. (3.19). When this is the case solutions of Eq. (3.29) exhibit finite time blow up [20]; the blow up time corresponds to the occurrence of a phase slip, i.e., the breakdown of the phase description. In numerical simulations, solutions of Eq. (1.1) always blow up owing to the generation of high frequency modes in this regime. This result is consistent with the failure of well-posedness when $\left|a_{1}-a_{2}\right|>2$. In figure 7 we present numerical results before blow up occurs. In contrast with the generic situation, $|\gamma|=O(1)>0$, the rolls narrow right before a phase slip.

\subsection{Amplitude and oscillatory modes}

We now assume that the periodic state satisfies $f=O(1)>0$ and that $g \approx 0$ (figure 2(b)). The instability sets in when $g$ becomes slightly negative and only occurs for periodic wavetrains slightly below the saddle node $\left(\mu_{0}=k_{0}^{2}-b^{\prime 2} / 4\right)$. The spectrum as a function of the perturbed wavenumber $q$ is shown in figure $2(\mathrm{~b})$ and indicates that the eigenvalues are real and unstable when $q$ is close to zero but become complex and unstable as $q$ exceeds a certain limit. Since

$$
f=f_{0} \equiv \frac{a_{2}^{2} b^{\prime 2}}{4}-\left(2 k_{0}+\frac{a_{1} b^{\prime}}{2}\right)^{2}
$$

when $\mu=\mu_{0}$, the coefficient $a_{2}$ must be nonzero and large enough for $f$ to be positive. Thus this type of instability is only present when the system is sufficiently far from a gradient system.

When $\mu$ is close to the onset of instability, the dependence of $g$ (Eq. (3.7)) on $\mu-\mu_{0}$ is

$$
g(\mu)=2\left(\mu-\mu_{0}\right)-b^{\prime} \sqrt{\mu-\mu_{0}} \approx-b^{\prime} \sqrt{\mu-\mu_{0}},
$$



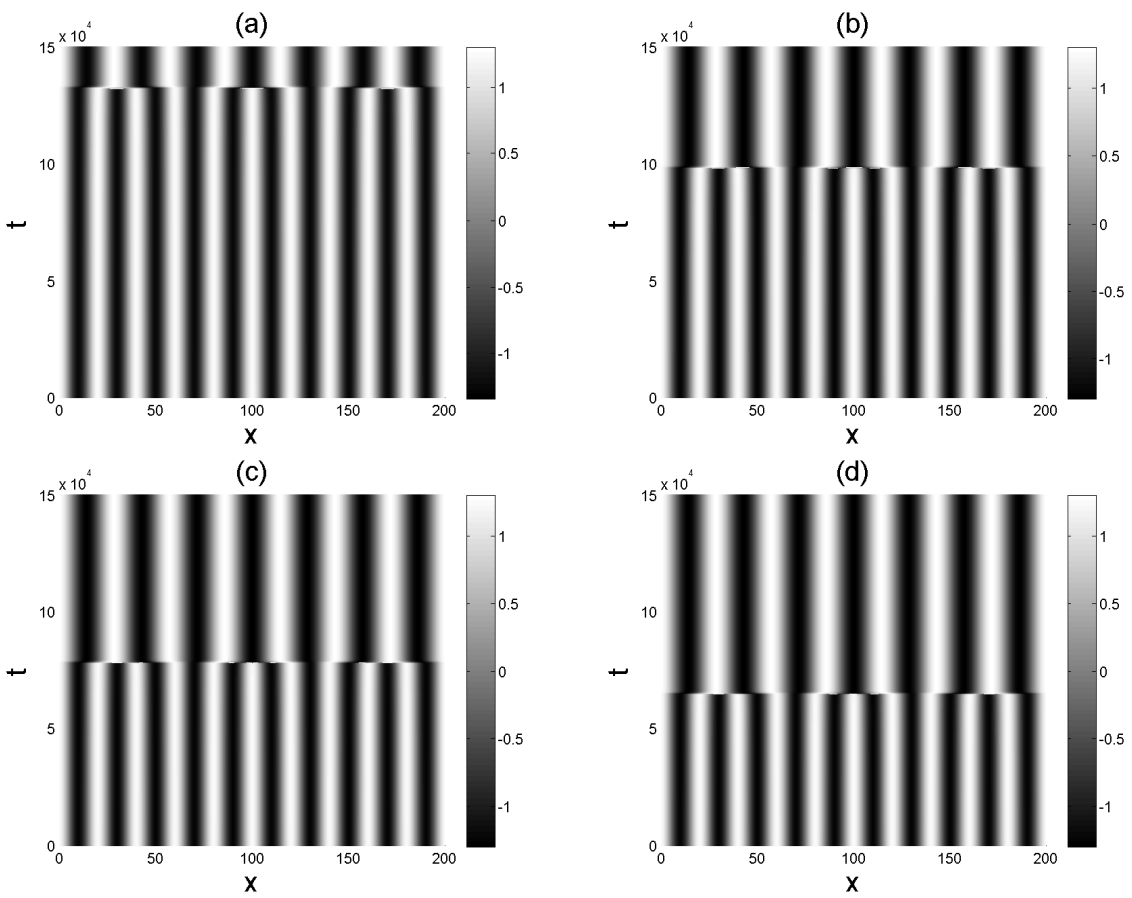

Figure 5. Time evolution of $\operatorname{Re}[A]$ with $\mathrm{PBC}$ in a domain of size $\Gamma=200$ triggered by the Eckhaus instability showing the effect of different initial growth rate. Parameter values: $a_{2}=0.2, g=1.3, \gamma=7$. (a) $f=-0.01503\left(\sigma_{+}=2.0847 \times 10^{-5}\right)$. $f=-0.01751\left(\sigma_{+}=2.9261 \times 10^{-5}\right)$. (c) $f=-0.01999\left(\sigma_{+}=3.7680 \times 10^{-5}\right)$.

$f=-0.02247\left(\sigma_{+}=4.6105 \times 10^{-5}\right)$. In each case the solution has been initialized with a sinusoidal perturbation of amplitude $\sqrt{c_{1}^{2}+c_{2}^{2}}=0.001$ and wavenumber $q=6 \pi / \Gamma$.
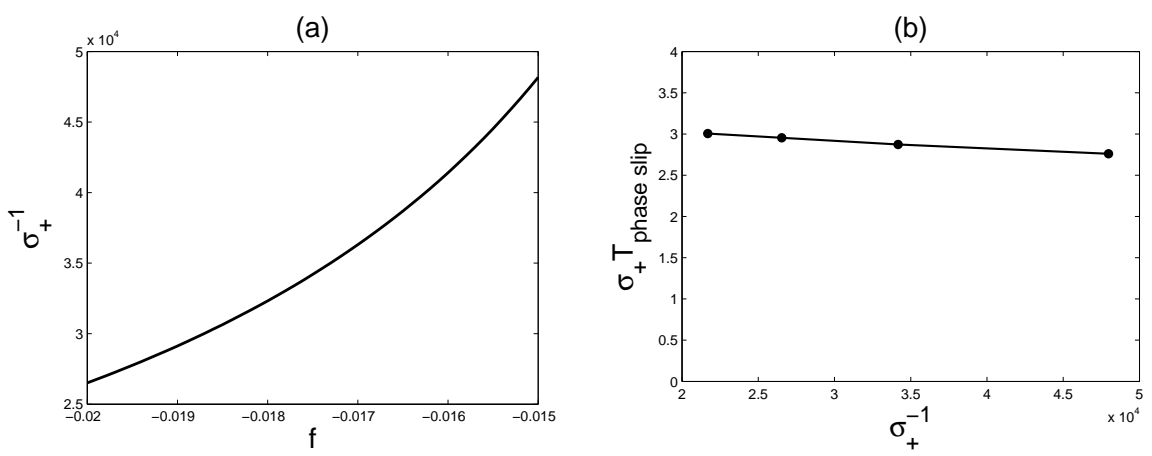

Figure 6. (a) The linear instability timescale $\sigma_{+}^{-1}$ as a function of $f$ from figure 5 . (b) Time $T$ to phase slip in figure 5 multiplied by $\sigma_{+}$as a function of $\sigma_{+}^{-1}$.

where $b^{\prime}$ is given by Eq. (3.2). Let $\delta \mu \equiv \mu-\mu_{0}=O\left(\epsilon^{4}\right)$. In order for $g$ to compete with the $q^{2}$ term in the temporal eigenvalue, the amplitude must vary on $O\left(\epsilon^{-1}\right)$ scales. But there is another spatial scale which comes from the modes with real unstable eigenvalues. These modes lie within a narrow region around $q=0$ of $O\left(\epsilon^{2}\right)$ width. The inclusion of this spatial scale is important when the domain size is relatively large. We refer to these modes as amplitude modes while the modes in the region of unstable complex eigenvalues are oscillatory modes. With the given scaling, the magnitudes of the real and the 


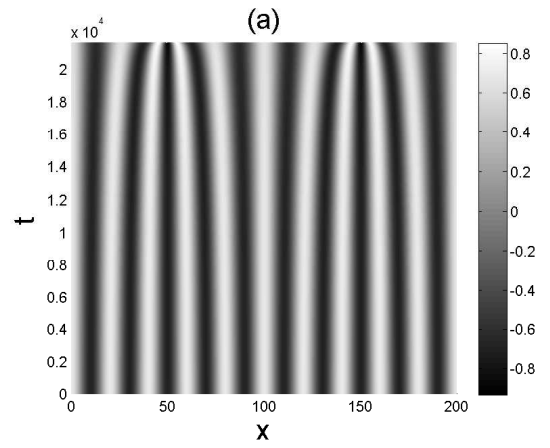

(b)

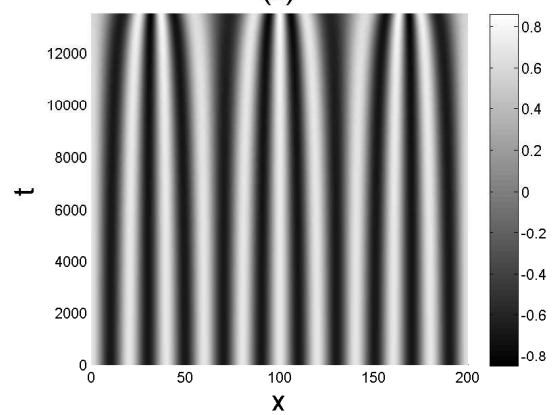

Figure 7. Time evolution of $\operatorname{Re}[A]$ with $\mathrm{PBC}$ in a domain of size $\Gamma=200$ prior to solution blow up. Parameter values: $a_{1}=-2.9, a_{2}=-0.7, f=-0.02, \gamma=0$. The instability is initialized using an unstable mode with wavenumber (a) $4 \pi / \Gamma$ and (b) $6 \pi / \Gamma$.

imaginary parts of the eigenvalues are of different order, $O\left(\epsilon^{-2}\right)$ for the real part and $O\left(\epsilon^{-1}\right)$ for the imaginary part. These facts suggest the following choice of spatial and temporal scales:

$$
\begin{aligned}
& X_{1}=\epsilon x, \quad X_{2}=\epsilon^{2} x, \quad T_{1}=\epsilon t, \quad T_{2}=\epsilon^{2} t, \\
& \delta \mu=\epsilon^{4} \mu_{1}^{2}, \quad a=\epsilon^{2} a^{(0)}+\epsilon^{3} a^{(1)}+\ldots
\end{aligned}
$$

Here $\delta \mu \geq 0$ since $\mu_{0}$ is already at the saddle node.

In the following we write $a^{(n)} \equiv a_{r}^{(n)}+i a_{i}^{(n)}$ and substitute the above Ansatz into Eq. (3.4). The first two orders in $\epsilon$ then yield the equations

$$
\begin{aligned}
& a_{r, T_{1}}^{(0)}-b_{-} a_{i, X_{1}}^{(0)}=0 \\
& a_{i, T_{1}}^{(0)}-b_{+} a_{r, X_{1}}^{(0)}=0 \\
& a_{r, T_{1}}^{(1)}-b_{-} a_{i, X_{1}}^{(1)}=b_{-} a_{i, X_{2}}^{(0)}-a_{r, T_{2}}^{(0)}+a_{r, X_{1} X_{1}}^{(0)}-b^{\prime 2} a_{r}^{(0) 2}+2 b^{\prime} \mu_{1} a_{r}^{(0)}, \\
& a_{i, T_{1}}^{(1)}-b_{+} a_{r, X_{1}}^{(1)}=b_{+} a_{r, X_{2}}^{(0)}-a_{i, T_{2}}^{(0)}+a_{i, X_{1} X_{1}}^{(0)} .
\end{aligned}
$$

Here $b_{ \pm}$refers to the quantities defined in Eq. (3.17), evaluated at $\mu=\mu_{0}$. Thus $a_{r}^{(0)}$ and $a_{i}^{(0)}$ both satisfy a one-dimensional wave equation on $O\left(\epsilon^{-1}\right)$ scales with wave speed $\sqrt{f_{0}}$ (Eq. (3.30)). We therefore introduce the comoving variables $\xi \equiv X_{1}-\sqrt{f_{0}} T_{1}$ and $\eta \equiv X_{1}+\sqrt{f_{0}} T_{1}$ and look for solutions of the form

$$
\begin{aligned}
& a_{r}^{(0)}=A_{1}\left(\xi, X_{2}, T_{2}\right)+A_{2}\left(\eta, X_{2}, T_{2}\right)+A_{0}\left(X_{2}, T_{2}\right), \\
& a_{i}^{(0)}=\left(b_{+} / b_{-}\right)^{1 / 2}\left[-A_{1}\left(\xi, X_{2}, T_{2}\right)+A_{2}\left(\eta, X_{2}, T_{2}\right)+\tilde{A}_{0}\left(X_{2}, T_{2}\right)\right] .
\end{aligned}
$$

Here $A_{0}$ and $\tilde{A}_{0}$ represent the effects of adjustment in the mean. Combining Eqs. (3.35) and (3.36) gives

$$
2 \sqrt{f_{0}} a_{r, \xi \eta}^{(1)}=\left(\partial_{T_{2}}-b^{\prime} \mu_{1}+b^{\prime 2} a_{r}^{(0)}\right)\left(A_{2, \eta}-A_{1, \xi}\right)+A_{1, \xi \xi \xi}-A_{2, \eta \eta \eta}-\sqrt{f_{0}}\left(A_{1, \xi}+A_{2, \eta}\right)_{X_{2}} .
$$

Requiring that $A_{1}$ and $A_{2}$ remain bounded as $|\xi| \rightarrow \infty,|\eta| \rightarrow \infty$, we obtain the following coupled equations for the slow evolution of the amplitudes $A_{1}$ and $A_{2}$,

$$
\begin{aligned}
& \left(\partial_{T_{2}}+\sqrt{f_{0}} \partial_{X_{2}}\right) A_{1, \xi}=A_{1, \xi \xi \xi}+b^{\prime} \mu_{1} A_{1, \xi}-b^{\prime 2}\left(A_{1}+A_{0}\right) A_{1, \xi} \\
& \left(\partial_{T_{2}}-\sqrt{f_{0}} \partial_{X_{2}}\right) A_{2, \eta}=A_{2, \eta \eta \eta}+b^{\prime} \mu_{1} A_{2, \eta}-b^{\prime 2}\left(A_{2}+A_{0}\right) A_{2, \eta} .
\end{aligned}
$$


Since the spatial average of $A_{1}$ and $A_{2}$ can be absorbed into $A_{0}$ and $\tilde{A}_{0}$, we may assume $\left\langle A_{1}\right\rangle=\left\langle A_{2}\right\rangle=$ 0 , where $\langle\cdot\rangle$ indicates the average over the comoving space variables. Integrating both Eqs. (3.40) and (3.41) once and averaging Eqs. (3.35) and (3.36) with respect to $\xi$ and $\eta$, respectively, we obtain finally a pair of equations that describe the interaction between the amplitude and oscillatory modes,

$$
\begin{aligned}
& \left(\partial_{T_{2}}+\sqrt{f_{0}} \partial_{X_{2}}\right) A_{1}=A_{1, \xi \xi}+\left(\mu_{1}-A_{0}\right) A_{1}-\frac{A_{1}^{2}-\left\langle A_{1}^{2}\right\rangle}{2}, \\
& \left(\partial_{T_{2}}-\sqrt{f_{0}} \partial_{X_{2}}\right) A_{2}=A_{2, \eta \eta}+\left(\mu_{1}-A_{0}\right) A_{2}-\frac{A_{2}^{2}-\left\langle A_{2}^{2}\right\rangle}{2}, \\
& \tilde{A}_{0, T_{2}}-\sqrt{f_{0}} A_{0, X_{2}}=0, \quad A_{0, T_{2}}-\sqrt{f_{0}} \tilde{A}_{0, X_{2}}=2 \mu_{1} A_{0}-\left\langle A_{1}^{2}+A_{2}^{2}\right\rangle-A_{0}^{2} .
\end{aligned}
$$

Here the coefficient $b^{\prime}$ has been scaled out by suitably redefining $A_{1}, A_{2}, A_{0}, \tilde{A}_{0}$, and $\mu_{1}$. These equations generalize those derived by Knobloch and De Luca for counterpropagating waves on a trivial background [22] to the case of a nontrivial background.

Figures 8 and 9 show plots of the time evolution of Eq. (1.1) in a periodic domain of size $\Gamma=200$ for $a_{1}=0.3, a_{2}=2.2, b=0.5$, and $\mu=\mu_{0}+5 \times 10^{-6}$. The initial 20 roll periodic wavetrain is perturbed with a right-traveling wave with $q=2 \pi / \Gamma$ (figure 8) or a left-traveling wave with $q=4 \pi / \Gamma$ (figure 9 ). Both perturbations are of order $10^{-2}$. Under these conditions, the wavetrain eventually collapses to 0 although a growing traveling modulation is visible in the demodulated solution as shown in figures $8(\mathrm{~b})$ and 9(b). A comparison between the dynamics of the full equation (1.1) and the modulation equations is shown in figure 8(c), which demonstrates that the time dependence of $\left(b^{\prime 2} / \epsilon^{2}\right) \operatorname{Re}[\langle a\rangle]$ (solid line) is well approximated by the time evolution of the mean mode $A_{0}$ (dashed line).
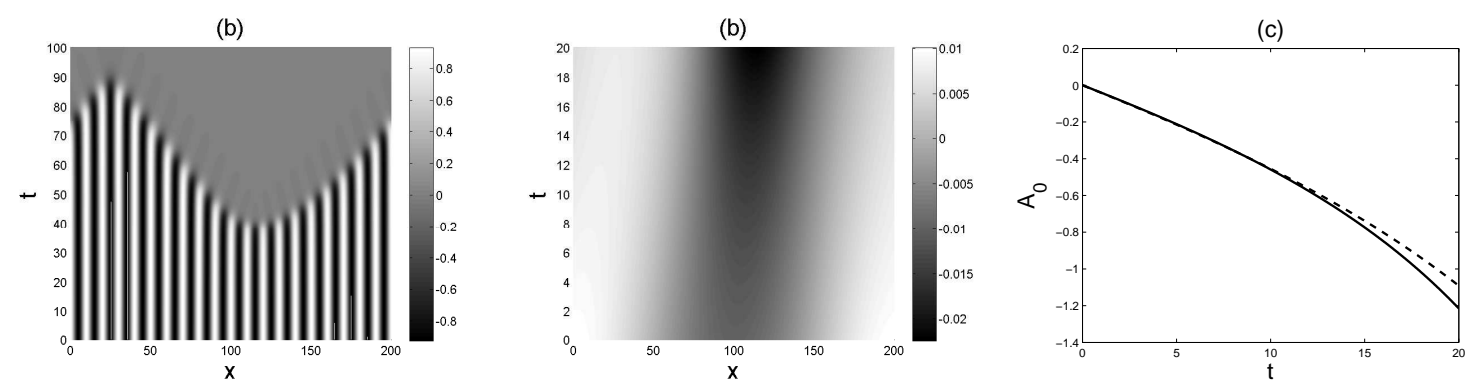

Figure 8. A 20 roll periodic wavetrain with PBC undergoing both amplitude and oscillatory instabilities. (a) Time evolution of $\operatorname{Re}[A]$. (b) Time evolution of $\operatorname{Re}[a]$ (relation $(3.3))$. (c) Comparison of the evolution of the mean of the modulation mode (solid line) with the evolution of $A_{0}$ from Eq. (3.44) (dashed line). Domain size: $\Gamma=200$. Parameter values: $a_{1}=0.3, a_{2}=2.2, b=0.5$, and $\mu=\mu_{0}+5 \times 10^{-6}$. The initial condition is a periodic wavetrain with a superposed $q=2 \pi / \Gamma$ right-traveling wave of $O\left(10^{-2}\right)$ amplitude.

\section{Stability and dynamics of spatially localized solutions near the Maxwell point}

In this section we study the stability and dynamics of spatially localized solutions near the Maxwell point. Dynamical properties of these solutions are important for understanding the location of the snaking region and the process of nucleation of periodic patterns in convective systems. 
(a)

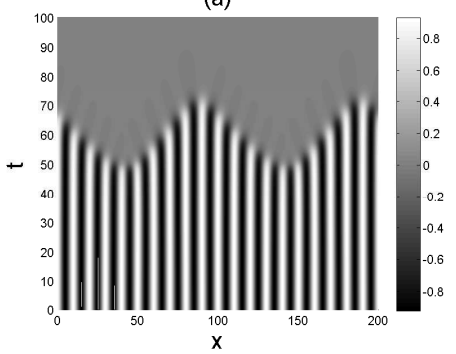

(b)

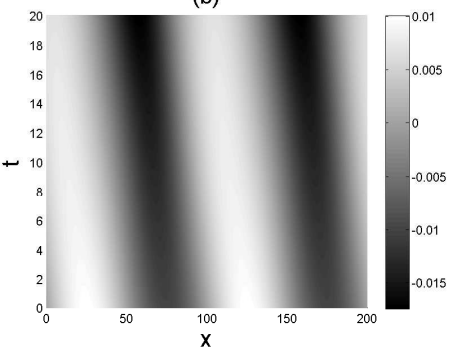

(c)

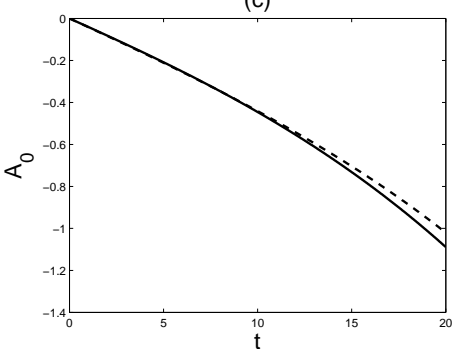

Figure 9. A 20 roll periodic wavetrain with PBC undergoing both amplitude and oscillatory instabilities. (a) Time evolution of $\operatorname{Re}[A]$. (b) Time evolution of $\operatorname{Re}[a]$ (relation $(3.3))$. (c) Comparison of the evolution of the mean of the modulation mode (solid line) with the evolution of $A_{0}$ from Eq. (3.44) (dashed line). Domain size: $\Gamma=200$. Parameter values: $a_{1}=0.3, a_{2}=2.2, b=0.5$, and $\mu=\mu_{0}+5 \times 10^{-6}$. The initial condition is a periodic wavetrain with a superposed $q=4 \pi / \Gamma$ left-traveling wave of $O\left(10^{-2}\right)$ amplitude.

\subsection{Stationary and nonstationary fronts}

Among the spatially localized solutions, the front solutions determine the location of the Maxwell point and the associated protosnaking behavior. With the coefficients $a_{1}, a_{2}$ and $b$ fixed, there is at most one stationary front solution to within the symmetries given in section 2 . This solution connects the trivial state $A=0$ to one of the periodic states. As shown in [1], the front solution exists when $E=L=0$ with coefficients $b>0, \mu=\mu_{M} \equiv b^{2} /(16 \beta)<0$. In order to simplify the discussion that follows, we set without loss of generality $b=1$ and obtain the explicit solution

$$
R^{2}=\frac{-4 \mu_{M}}{e^{ \pm 2 \sqrt{-\mu_{M}} x}+1}, \quad \phi_{x}=\frac{\left(a_{1}+a_{2}\right) \mu_{M}}{e^{ \pm 2 \sqrt{-\mu_{M}} x}+1}
$$

The selected wavenumber $k_{M}$ at the Maxwell point is given by $k_{M}=\left(a_{1}+a_{2}\right) \mu_{M}$.

The front solution can only be stable when the asymptotic states as $x \rightarrow \pm \infty$ are both stable and no unstable point eigenvalue is present [11]. The same argument also applies to other spatially extended solutions. Since $\mu$ is negative, the zero asymptotic state is always linearly stable. The stability of the competing periodic state is determined by the sign of the coefficient $g$, here given by $g=\frac{8 \mu_{M}^{2}}{3}\left[4-a_{2}\left(a_{1}+a_{2}\right)\right]$. Thus the competing periodic state is unstable when $a_{2}\left(a_{1}+a_{2}\right)>4$ and stable when $a_{2}\left(a_{1}+a_{2}\right)<4$. To check for the existence of unstable point eigenvalues when $g>0$, we solve an eigenvalue problem analogous to Eq. (3.4), viz.

$$
\begin{aligned}
a_{t}= & a_{x x}+\left[2 R^{-1} R_{x}+i\left(\frac{a_{1}-a_{2}}{2} R^{2}+\frac{2 L}{R^{2}}\right)\right] a_{x}+i a_{2} R^{2} a_{x}^{*} \\
& +\left[b R^{2}+\left(\frac{a_{1}^{2}-a_{2}^{2}}{4}-2\right) R^{4}+\left(a_{2}-a_{1}\right) L+i\left(a_{1}+a_{2}\right) R R_{x}\right]\left(a+a^{*}\right) .
\end{aligned}
$$

We solve this problem numerically using the Ansatz $a(x, t)=(u+v) e^{\sigma t}+\left(u^{*}-v^{*}\right) e^{\sigma^{*} t}$, where $u$ and $v$ are functions of $x$. The resulting eigenvalue problem takes the form

$$
\sigma\left(\begin{array}{l}
u \\
v
\end{array}\right)=\left(\begin{array}{cc}
\partial_{x x}+A \partial_{x}+C & i\left(B-a_{2} R^{2}\right) \partial_{x} \\
i\left(B+a_{2} R^{2}\right) \partial_{x}+i D & \partial_{x x}+A \partial_{x}
\end{array}\right)\left(\begin{array}{l}
u \\
v
\end{array}\right) .
$$


where

$$
\begin{aligned}
& A=2 R_{x} / R, \quad B=\frac{a_{1}-a_{2}}{2} R^{2}+\frac{2 L}{R^{2}} \\
& C=2 b R^{2}+\left(\frac{a_{1}^{2}-a_{2}^{2}}{2}-4\right) R^{4}+2\left(a_{2}-a_{1}\right) L, \quad D=\left(a_{1}+a_{2}\right)\left(R^{2}\right)_{x} .
\end{aligned}
$$

The temporal stability of the front solution depends on the coefficients $a_{1}$ and $a_{2}$; since $L=0$ the stability of the solution with coefficients $\left(a_{1}, a_{2}\right)$ is identical to that with coefficients $\left(-a_{1},-a_{2}\right)$. The problem is solved using a spectral method with cosine and sine basis functions with wavenumbers equal to integer multiples of $\pi / \Gamma$, where $\Gamma=50 / \sqrt{-\mu_{M}}$. The spectral matrix is truncated after the first 512 modes. The same procedure is also used for the stability calculation for the other spatially localized solutions examined below. The numerical results indicate that there is no unstable point eigenvalue for solutions with stable asymptotic states within the range $\left|a_{1}\right|,\left|a_{2}\right| \leq 10$, suggesting that this family of solutions is linearly stable. This result holds independently of the value of $b$ provided only that $b>0$.

Motion of the stable front can be generated by shifting the parameter $\mu$ slightly from the Maxwell point, i.e., by introducing a difference in "energy" between the two competing states. The existence of these moving front solutions has only been proved in the case $\left|a_{1}+a_{2}\right| \ll 1$ [9] but our numerical simulations indicate that these traveling front solutions continue to exist when $\left|a_{1}+a_{2}\right|=O(1)$. When $\mu<\mu_{M}$ the trivial state has the lower "energy" and the front therefore moves to eliminate the periodic wavetrain. The opposite occurs for $\mu>\mu_{M}$. The time evolution of such moving fronts with coefficients $a_{1}=1, a_{2}=1$ is shown in figure 10. Here we give the details of an asymptotic calculation of the resulting front speed when $\left|\mu-\mu_{M}\right| \ll 1$.

(a)

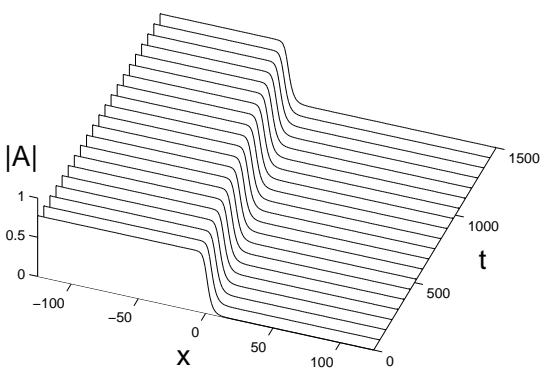

(b)

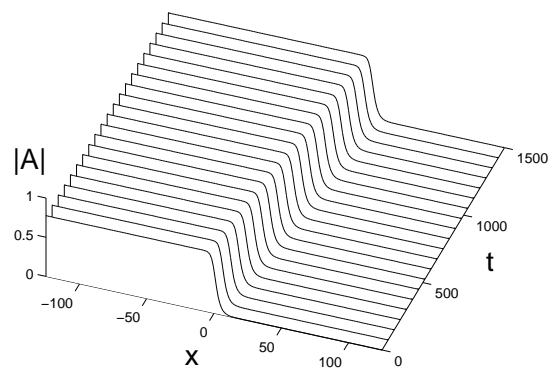

Figure 10. Time evolution of the profile of a stable front with $\mu$ perturbed from $\mu_{M}$. Parameter values: $a_{1}=1, a_{2}=1, b=1$. (a) A retracting front: $\mu=1.01 \mu_{M}$. (b) An advancing front: $\mu=0.99 \mu_{M}$.

Equation (1.1), written in terms of $R$ and $k \equiv \phi_{x}$, takes the form

$$
\begin{aligned}
& R_{t}=\left(\mu-k^{2}\right) R+R_{x x}+\left[b+k\left(a_{2}-a_{1}\right)\right] R^{3}-R^{5}, \\
& k_{t}=\left(k+\frac{a_{1}+a_{2}}{2} R^{2}\right)_{x x}+\left(\frac{2 k R_{x}}{R}\right)_{x} .
\end{aligned}
$$

According to the numerical results, both $R$ and $k$ drift slowly with a certain speed $c$. In the comoving frame, we have

$$
\begin{aligned}
& -c R_{x}=\left(\mu-k^{2}\right) R+R_{x x}+\left[b+k\left(a_{2}-a_{1}\right)\right] R^{3}-R^{5}, \\
& -c k_{x}=\left(k+\frac{a_{1}+a_{2}}{2} R^{2}\right)_{x x}+\left(\frac{2 k R_{x}}{R}\right)_{x},
\end{aligned}
$$


where $x$ now refers to the space variable in the comoving frame. Equation (4.6) can also be written as

$$
-c R_{x}=R_{x x}+\frac{1}{2} U_{R}
$$

where $U$ is defined in Eq. (2.4). Integrating Eq. (4.7) once and multiplying it by $R^{2}$ leads to

$$
L_{x}=R^{2} c\left(k_{\infty}-k\right),
$$

where $k_{\infty}$ is the wavenumber of the periodic state as $x \rightarrow-\infty$. Assuming that the trivial solution is present at $x \rightarrow \infty$ we have

$$
L=c \int_{x}^{\infty} R^{2}\left(k-k_{\infty}\right) d x
$$

indicating that $L$ is proportional to the speed of the front $c$, at least when $c$ is small. Let $R=R_{f}+r$, where $R_{f}$ is the amplitude of the stationary front solution and $r$ is a small perturbation. Substituting $R=R_{f}+r$ into Eq. (4.8) and collecting leading order terms yields

$$
-c R_{f, x} \approx\left(\mu-\mu_{M}\right) R_{f}+\frac{3 a_{2}-a_{1}}{2} L R_{f}+r_{x x}+\frac{1}{2} U_{R R}\left(R_{f} ; \mu_{M}, 0\right) r .
$$

Multiplying this equation by $-2 R_{f, x}$ and integrating over the spatial domain we obtain

$$
\left.2 c \int_{\mathbb{R}} R_{f, x}^{2} d x \approx\left(\mu-\mu_{M}\right) R_{f}^{2}\right|_{x=-\infty}+\left(a_{1}-3 a_{2}\right) \int_{\mathbb{R}} L R_{f} R_{f, x} d x .
$$

The contribution of the terms proportional to $r$ vanishes since $R_{f, x}$ corresponds to the infinitesimal translation mode in the stationary case. After evaluating the integrals we have

$$
c \approx \frac{3\left(\mu-\mu_{M}\right)}{2\left(-\mu_{M}\right)^{3 / 2}}\left[4-a_{2}\left(a_{1}+a_{2}\right)\right]^{-1} .
$$

This expression reduces to the front speed as calculated in [9] when $a_{1}+a_{2}=0$. The coefficient in the denominator is always positive for stable stationary front solutions and the sign of $c$ is then in agreement with the energy argument. The approximate front speed agrees well with the numerical results when $\mu$ is close to $\mu_{M}$ as shown in figure 11 .

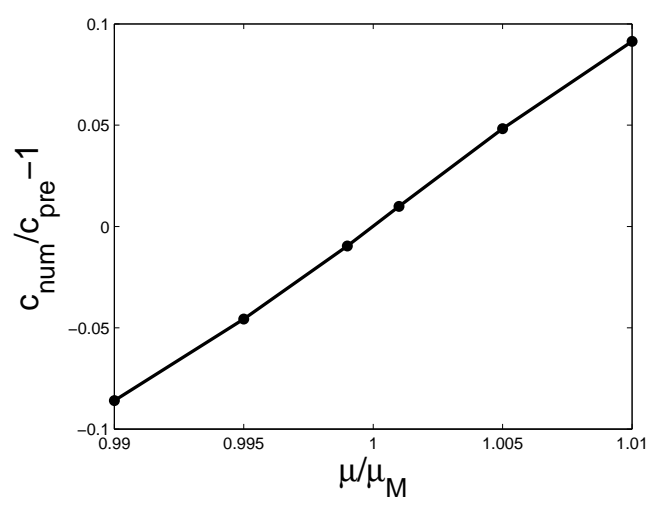

FiguRE 11 . The quantity $c_{\text {num }} / c_{\text {pre }}-1$ as a function of $\mu / \mu_{M}$ for front solutions when $a_{1}=a_{2}=1$. Here $c_{n u m}$ is the measured speed and $c_{\text {pre }}$ is the predicted value. 
For unstable fronts, we perform numerical simulations initialized with the stationary front solution and a superposed small amplitude localized perturbation at the center. Since the unstable part of the front lies in between the stable trivial state and stable periodic state, the perturbation can either grow or decay, depending on the details of the initial condition. If the front solution is initially slightly suppressed at the center, simulations reveal that the adjacent unstable part collapses, allowing the trivial state to invade the unstable state. Figure 12(a) shows this behavior when $a_{1}=2, a_{2}=3$; the speed of the resulting front is approximately 1.14. In figure 12(b), the coefficients used are the same as in figure $12(\mathrm{a})$ but the initial profile is slightly augmented at the center instead. This perturbation leads to the growth of an unstable periodic state followed by a transition to a stable periodic state which then invades both the stable zero state and the unstable periodic state. The measured front speed between the stable and unstable periodic states is approximately 1.27 while that between stable periodic state and $A=0$ is approximately 0.24 .

In all the simulations domain truncation has been applied. In order to implement suitable boundary conditions we compute the dynamical behavior of $\tilde{A}(x, t) \equiv A(x, t) e^{-i k_{M} x}$, where $k_{M} \equiv\left(a_{1}+a_{2}\right) \mu_{M}$ is the asymptotic wavenumber as $x \rightarrow \pm \infty$ when $\mu=\mu_{M}$, instead of dealing with $A$. Thus $\tilde{A}$ tends to a constant as $x \rightarrow-\infty$ and this constant can be chosen to be a real number. Cosine and sine functions with wavenumbers equal to integer multiples of $\pi / \Gamma$ are used as basis functions. A similar method is also used in the simulation of the dynamics of perturbed hole and pulse solutions discussed below.

(a)

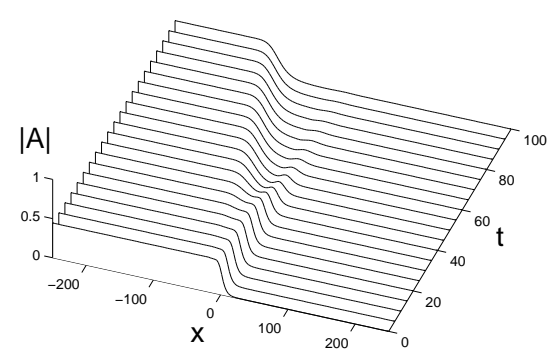

(b)

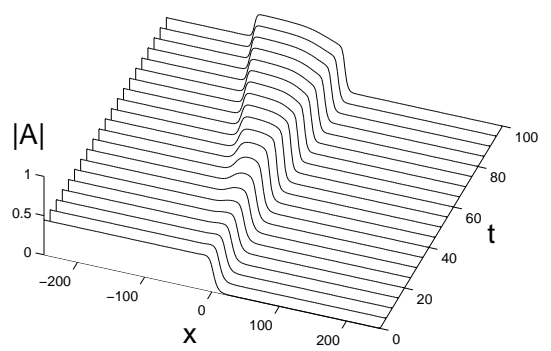

Figure 12. Time evolution of the profile of an unstable front. Parameter values: $a_{1}=2$, $a_{2}=3, b=1$, and $\mu=-0.0492$. The center of the front is suppressed by a factor 0.99 in (a) and augmented by 1.01 in (b).

The front motion presented in figure 12(a) is an example of front propagation into an unstable state. In many cases the speed of the resulting front is selected by the marginal stability condition [16,17]. This prediction focuses on modes of the form

$$
a_{\tilde{q}, \lambda} \sim \beta_{1} e^{i \tilde{q} x}+\beta_{2}^{*} e^{-i \tilde{q}^{*} x}
$$

where $\tilde{q}$ is now a complex wavenumber, $\tilde{q} \equiv q+i \lambda$. Thus $\lambda$ represents the spatial decay or growth rate of the mode. This mode has the dispersion relation $\sigma_{ \pm}(\tilde{q})$, where $\sigma_{ \pm}$is given in Eq. (3.5). In the present case it is the + branch of the dispersion relation that is relevant. The linear spreading speed is thus given by the relation

$$
v^{*} \equiv i \frac{d \sigma_{+}}{d \tilde{q}}=\frac{\operatorname{Re}\left[\sigma_{+}(\tilde{q})\right]}{\lambda},
$$

or equivalently

$$
v^{*}=\min _{\lambda} \max _{q}\left\{\frac{\operatorname{Re}\left[\sigma_{+}(\tilde{q})\right]}{\lambda}\right\} .
$$


Pinch point analysis in the complex $\tilde{q}$ plane [17] now leads to the prediction

$$
q=0, \quad \lambda^{* 2}=\frac{g}{2(2 g-f)}\left(2 f-3 g+\sqrt{9 g^{2}-4 f g}\right),
$$

and hence, from Eq. (4.16), to the explicit result

$$
v^{*}=\frac{\lambda^{*}}{1+g / \lambda^{* 2}}
$$

For the parameter values of figure 12 this expression predicts that $v^{*}=1.1642$, compared with the measured speeds $v^{*}=1.14$ in figure $12(\mathrm{a})$ and $v^{*}=1.27$ in figure $12(\mathrm{~b})$. Moreover, since $q=0$ the wavenumber $k^{*}$ selected by the invading front is [16]

$$
k^{*} \equiv \frac{\operatorname{Im}\left[\sigma_{+}(\tilde{q})+i \tilde{q} v^{*}\right]}{v^{*}}=0 .
$$

Figure 13 shows these results in the $\left(a_{1}, a_{2}\right)$ plane.

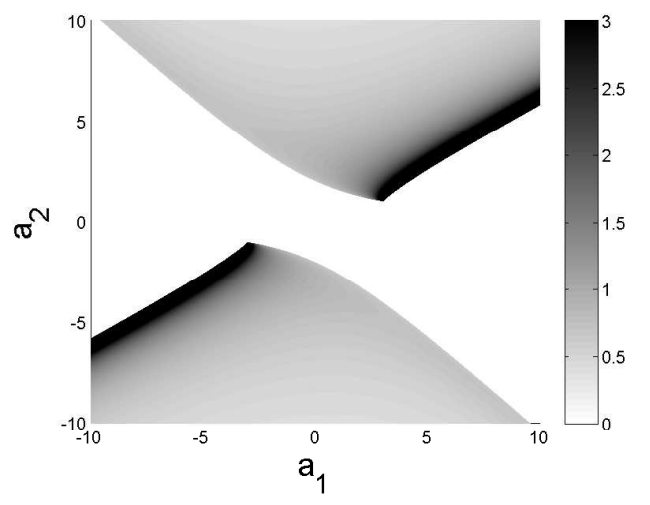

Figure 13. The linear spreading speed of the unstable front solution in the $\left(a_{1}, a_{2}\right)$ plane. As $\left(a_{1}, a_{2}\right)$ approaches the line $\beta=0$ (Eq. (2.6)), $v^{*}$ diverges. We therefore cut off $v^{*}$ at $v^{*}=3$ and use black color to indicate the parameter range where $v^{*} \geq 3$. In addition, $v^{*}$ is set equal to 0 in regions where either $\beta$ or $g$ is nonnegative (white color).

Note that figure 12(b) shows a second front as well, in which the stable large amplitude state invades the stable trivial state $A=0$. The speed of this type of front cannot be obtained from the linear stability analysis described above [17].

\subsection{Stationary holes and pulses}

Equation (1.1) possesses several other spatially localized solutions of interest, in addition to the front solutions just described. We focus here on the case $b=1, \beta<0$ for which the potential energy $U(R, L=0)$ takes the form shown in figure 14. Thus hole solutions exist for $\mu$ both greater and less than $\mu_{M}$.

When $\mu>\mu_{M}$ the holes take the form

$$
R^{2}=\frac{\xi_{1} \xi_{2} \sinh ^{2}\left(\sqrt{\mu+\frac{\xi_{1}}{2}} x\right)}{\xi_{2} \cosh ^{2}\left(\sqrt{\mu+\frac{\xi_{1}}{2}} x\right)-\xi_{1}}
$$


(a)

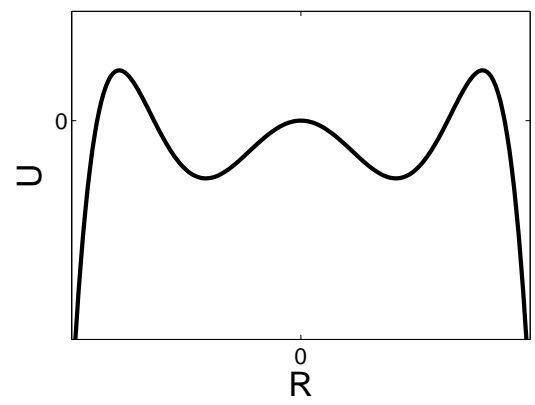

(b)

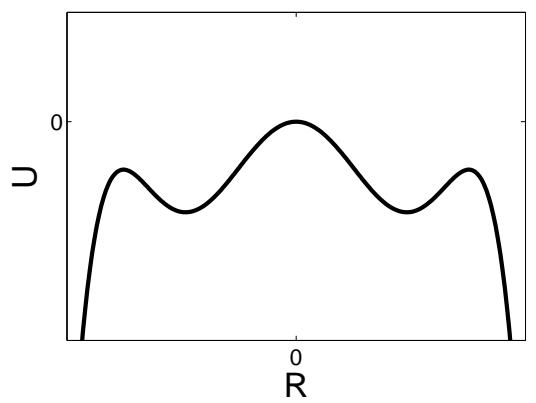

Figure 14. The potential energy $U\left(R, L=0\right.$ ) when (a) $\mu>\mu_{M}$ and (b) $\frac{4 \mu_{M}}{3}<\mu<\mu_{M}$.

with asymptotic wavenumber $k_{\infty}=-\left(a_{1}+a_{2}\right) \xi_{1} / 4$ as $x \rightarrow \pm \infty$, where

$$
\xi_{1}=-\frac{4 \mu_{M}}{3}\left(2+\sqrt{4-\frac{3 \mu}{\mu_{M}}}\right), \quad \xi_{2}=\frac{8 \mu_{M}}{3}\left(-1+\sqrt{4-\frac{3 \mu}{\mu_{M}}}\right) .
$$

The region of stability of these solutions in parameter space is shown in figure 15 for the case $\mu=$ $0.99 \mu_{M}>\mu_{M}$ and indicated by red (or gray) color. Most of the solutions with stable asymptotic states are stable except those with $\beta \approx 0$, as indicated in the figure. Figure 16 shows the dynamical evolution in the unstable case $\left(a_{1}, a_{2}\right)=(2,0)$, starting from an initial condition with $L=0$. For these coefficients the asymptotic states $x \rightarrow \pm \infty$ are stable but the hole state is nevertheless unstable. To reveal the instability the amplitude $|A|$ in a small interval around the center of the hole has been multiplied by a factor 0.99 in (a) and by 1.01 in (b), and the results used as initial conditions in a time evolution code. In the former case the solution converges quickly to a nearby stationary hole solution with $L \neq 0$. In the latter case the solution relaxes to the stable asymptotic state.

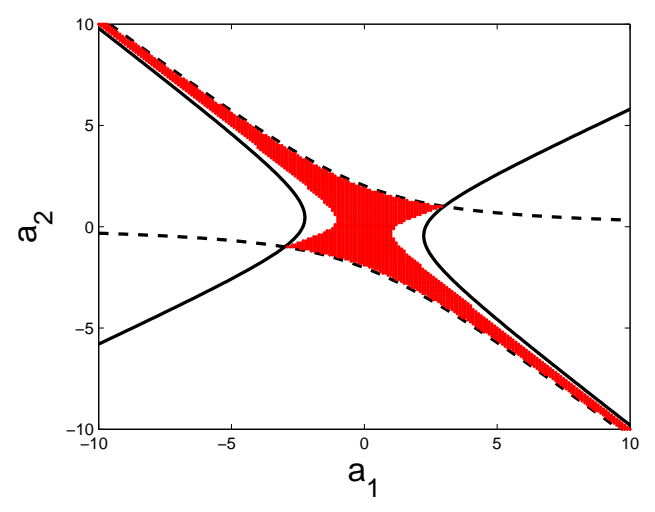

Figure 15. (Color online) The case $\mu=0.99 \mu_{M}$. Solid line: $\beta=0$. Dashed line: $g=0$. The quantity $\beta$ is negative between the solid lines while $g$ is positive between the dashed lines. Hole solutions are stable in the red (or gray) region. 
(a)

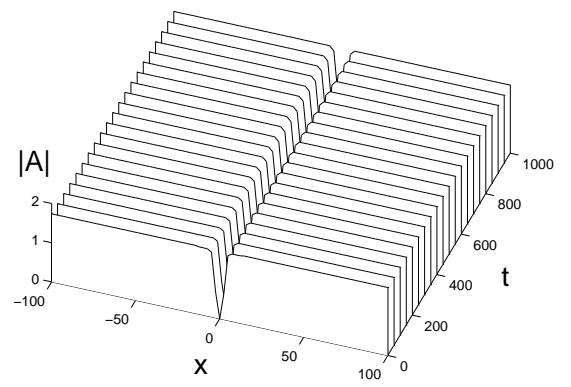

(b)

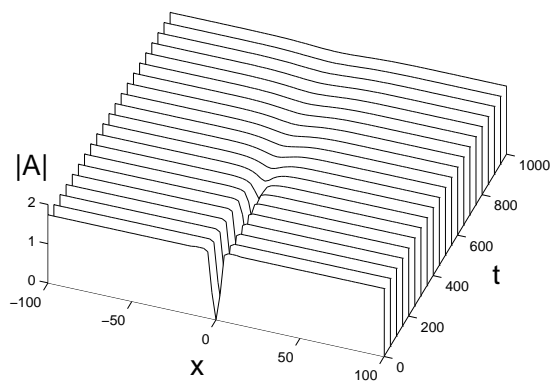

Figure 16. Dynamics of an unstable $L=0$ hole solution when $\mu=0.99 \mu_{M}$ and $\left(a_{1}, a_{2}\right)=(2,0)$. The amplitude at center of the hole is multiplied by a factor 0.99 in (a) and by 1.01 in (b). In (a) the hole evolves to a nearby hole with $L \approx 0.01$ and nonzero amplitude at the center; in (b) the hole disappears.

When $\frac{4 \mu_{M}}{3}<\mu<\mu_{M}$ the stationary hole solutions take the form

$$
R^{2}=\frac{\xi_{1} \xi_{2} \cosh ^{2}\left(\sqrt{\mu+\frac{\xi_{1}}{2}} x\right)}{\xi_{1}+\xi_{2} \sinh ^{2}\left(\sqrt{\mu+\frac{\xi_{1}}{2}} x\right)} .
$$

Here $\xi_{1}$ and $\xi_{2}$ are as defined in Eq. (4.21) and the asymptotic wavenumber remains $k_{\infty}=-\left(a_{1}+a_{2}\right) \xi_{1} / 4$. The region of stability of these hole solutions in parameter space is shown in figure 17 for the case $\mu=1.01 \mu_{M}<\mu_{M}$ and indicated by red (or gray) color. In contrast with the case $\mu>\mu_{M}$, almost all of the solutions are now unstable except for those with $\beta \approx 0$. Figure 18 shows the dynamical evolution in the unstable case $\left(a_{1}, a_{2}\right)=(1,1)$ with stable asymptotic states. To generate the initial condition the amplitude at the center of the hole has been multiplied by a factor 0.99 in (a) and by 1.01 in (b). If the solution is initially suppressed at the center the central part collapses to zero and invades the periodic structure in both directions as expected. However, if the solution is initially augmented at the center the amplitude at the central part overshoots the amplitude of the periodic state but then decays back. This behavior is a consequence of the non-gradient nature of the system when $a_{2} \neq 0$.

Pulses connecting the trivial state to itself exist only when $\mu_{M}<\mu<0$. These solutions take the form

$$
R^{2}=\frac{\xi_{1} \xi_{2}}{\xi_{1}+\left(\xi_{2}-\xi_{1}\right) \cosh ^{2}(\sqrt{-\mu} x)},
$$

where

$$
\xi_{1}=-4 \mu_{M}\left(1-\sqrt{1-\frac{\mu}{\mu_{M}}}\right), \quad \xi_{2}=-4 \mu_{M}\left(1+\sqrt{1-\frac{\mu}{\mu_{M}}}\right) .
$$

All such solutions appear to be unstable when $\mu=0.99 \mu_{M}$, at least within $\left|a_{1}\right|,\left|a_{2}\right| \leq 10$. Dynamical evolution of the unstable case $\left(a_{1}, a_{2}\right)=(1,1)$ is shown in figure 19. Depending on the perturbation applied initially, the solution either collapses to $A=0$ or broadens into a periodic state.

In the previous discussion we focused on localized states with $L=0$. Also of interest is the case when $k_{\infty}$, the asymptotic wavenumber of the localized state as $x \rightarrow \pm \infty$, is equal to $k_{M}$, the wavenumber at $\mu=\mu_{M}$. When $\mu$ is perturbed from $\mu_{M}, L$ becomes nonzero but remains small. The only stationary spatially localized states allowed are holes and these take the explicit form

$$
R^{2}=\xi_{1}+\frac{\left(\xi_{2}-\xi_{1}\right)\left(\xi_{3}-\xi_{1}\right)}{\xi_{2}-\xi_{1}+\left(\xi_{3}-\xi_{2}\right) \cosh ^{2}\left[\sqrt{\beta\left(\xi_{3}-\xi_{1}\right)\left(\xi_{1}-\xi_{2}\right)} x\right]},
$$




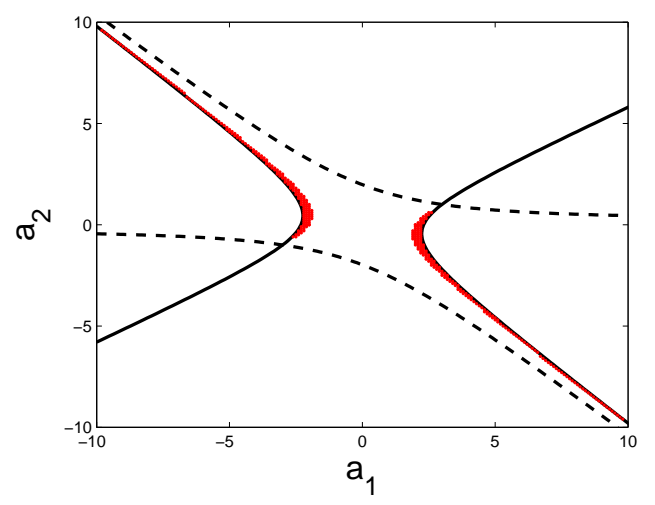

Figure 17. (Color online) The case $\mu=1.01 \mu_{M}$. Solid line: $\beta=0$. Dashed line: $g=0$. The quantity $\beta$ is negative between the solid lines while $g$ is positive between the dashed lines. Hole solutions are stable in the red (or gray) region.

(a)

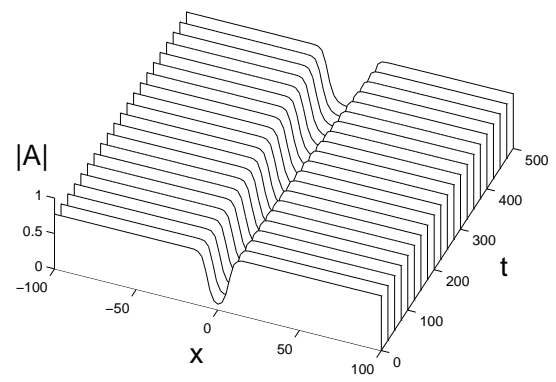

(b)

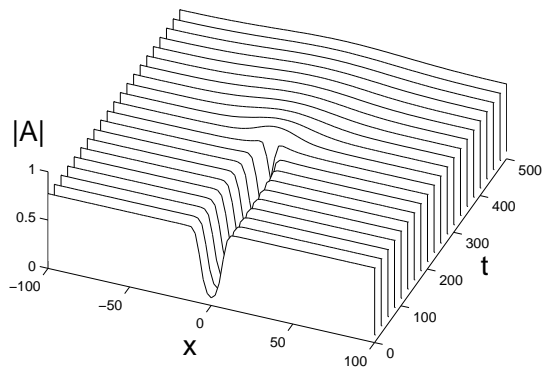

FiguRE 18. Dynamics of an unstable $L=0$ hole solution when $\mu=1.01 \mu_{M}$ and $\left(a_{1}, a_{2}\right)=(1,1)$. The amplitude at the center of the hole has been multiplied by a factor 0.99 in (a) and by 1.01 in (b). In (a) the hole expands; in (b) the hole decays.

where $\xi_{1}$ is the square of the amplitude of the asymptotic periodic state, $\xi_{2}$ is the square of the minimum amplitude of the solution, and $\xi_{3} \neq \xi_{1}, \xi_{2}$ is the remaining root of the polynomial $\xi[E-U(\sqrt{\xi})]$. We computed the stability properties of these solutions at $\mu=1.01 \mu_{M}$ and $0.99 \mu_{M}$ and found no stable solution when $\mu=1.01 \mu_{M}$ and $\left|a_{1}\right|,\left|a_{2}\right| \leq 10$. However, the solutions within $\left|a_{1}\right|,\left|a_{2}\right| \leq 10$ are stable when $\mu=0.99 \mu_{M}$.

To examine the dynamics that result from the instability, we set $\mu=1.01 \mu_{M}$ and choose an unstable solution with $\left(a_{1}, a_{2}\right)=(1,1)$. The resulting time evolution is shown in figure 20 and resembles that in figure 18 in which the solution either collapses to $A=0$ or approaches a periodic state in an oscillatory fashion. When $\mu=0.99 \mu_{M}$, we choose $\left(a_{1}, a_{2}\right)=(1,1.5)$; the resulting time evolution is shown in figure 21. This time the perturbation applied at the center is larger and generates oscillatory behavior near the center but the oscillation decays as time increases and the solution converges to the unperturbed solution.

\section{Conclusions}

In this paper we have explored, both analytically and numerically, the stability and dynamics of periodic wavetrains and spatially localized states in GL35 in the vicinity of the Maxwell point. The periodic 
(a)

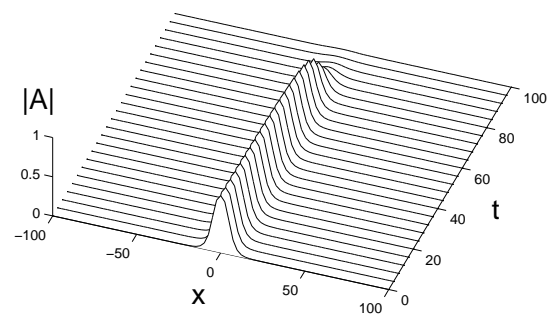

(b)

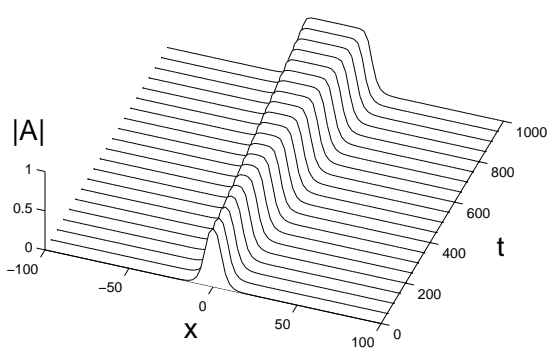

Figure 19. Dynamics of an unstable $L=0$ pulse solution when $\mu=0.99 \mu_{M}$ and $\left(a_{1}, a_{2}\right)=(1,1)$. The amplitude at the center of the hole has been multiplied by a factor 0.99 in (a) and by 1.01 in (b). In (a) the pulse decays to the flat state $A=0$; in (b) the pulse broadens into a periodic state.

(a)

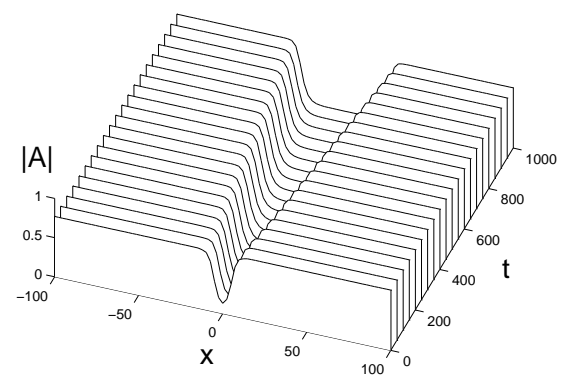

(b)

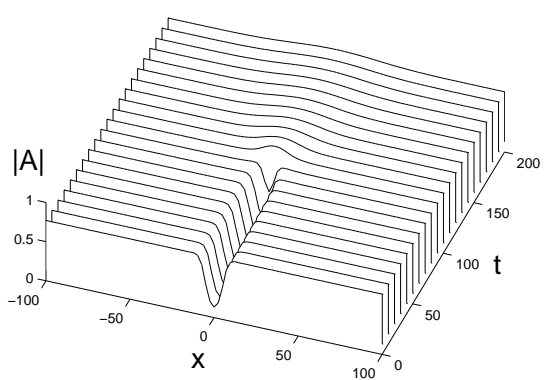

Figure 20. Evolution of an unstable hole solution with $k_{\infty}=k_{M}$ when $\mu=1.01 \mu_{M}$ and $\left(a_{1}, a_{2}\right)=(1,1)$. The amplitude at the center of the hole has been multiplied by a factor 0.99 in (a) and by 1.01 in (b). In (a) the hole broadens into a growing region filled with the flat state $A=0$; in (b) the hole decays into a constant amplitude periodic state.

wavetrains become unstable through two different mechanisms. One is the result of an unstable phase mode (Eckhaus instability) while the other is a consequence of an interaction between amplitude and oscillatory modes. In the former case we examined the bifurcations to spatially localized solutions within appropriate phase equations and investigated the evolution of the instability using direct numerical integration of GL35. The phase equation description breaks down at phase slips whereby the wavelength of the solution changes abruptly but coarsening of the structure may take place prior to this event. In the latter case the instability is oscillatory and the evolution takes the form of waves. The evolution of these waves is described by a pair of equations of Fisher-Kolmogorov type for the amplitudes of the left/right traveling waves coupled to an amplitude mode.

A systematic study of different spatially localized solutions was also performed. Solutions homoclinic to the trivial state (pulses) or to a periodic state (holes) were found near the analog of a Maxwell point at which a heteroclinic connection (front) between the trivial and periodic states forms. The formation of such a front is associated with protosnaking behavior as described in [1]. We have found that such fronts are stable when the asymptotic states are stable and vice versa. Away from the Maxwell point the fronts persist but move with a speed that depends on the distance from the Maxwell point. When the periodic state is unstable a wave of constant form can nonetheless be present and takes the form of 
(a)

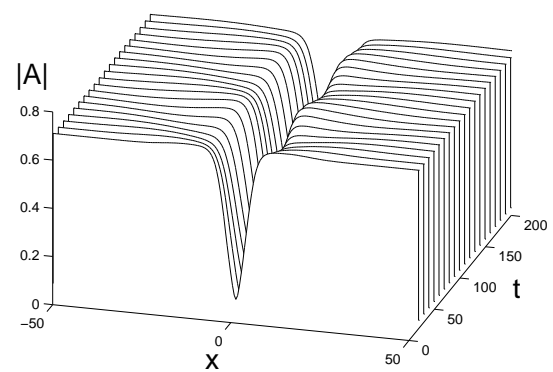

(b)

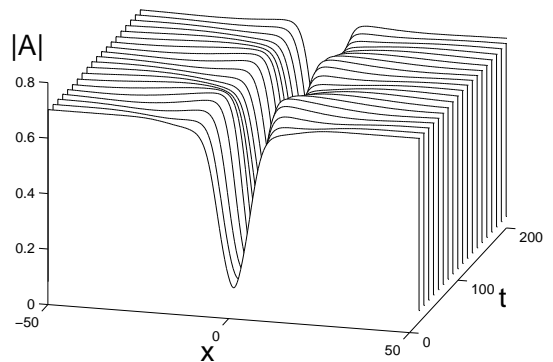

FIgURE 21. Evolution of a stable hole solution with $k_{\infty}=k_{M}$ when $\mu=0.99 \mu_{M}$ and $\left(a_{1}, a_{2}\right)=(1,1.5)$. The amplitude at the center of the hole has been multiplied by a factor 0.9 in (a) and by 1.1 in (b). The oscillations decay with increasing time.

an invasion front whereby a stable state invades an unstable state. We have computed the speed of such invasion fronts assuming that this speed is selected by the marginal stability criterion and showed that the resulting speed is in reasonable agreement with speeds measured in direct simulations of GL35. Stability properties of analytically determined pulse-like and hole-like solutions were determined numerically and in each case the stability region in the $\left(a_{1}, a_{2}\right)$ plane was determined.

The presence of the coefficients $a_{1}$ and $a_{2}$ in the GL35 is responsible for a variety of new and interesting phenomena, many due to the loss of variational structure when $a_{2} \neq 0$. These coefficients are readily derivable from systems at the transition between subcritical to supercritical bifurcation as shown in Appendix A for the Swift-Hohenberg equation. As a result the present work can be used to predict and classify the stationary and dynamical properties of all such systems. Related but nonlocal equations describe two-dimensional rotating convection with stress-free boundary conditions [21] and convection in an imposed vertical magnetic field. The corresponding behavior of such nonlocal amplitude equations is thus of considerable interest and will be the subject of a future publication.

\section{A. The generalized Swift-Hohenberg equation near the subcritical to supercritical transition}

We start from the generalized Swift-Hohenberg equation

$$
u_{t}=r u-\left(1+\partial_{x x}\right)^{2} u+f(u)
$$

where $u=u(x, t)$ is a real-valued function and $f(u)$ is a smooth function with Taylor expansion

$$
f(u)=f_{2} u^{2}+f_{3} u^{3}+f_{4} u^{4}+f_{5} u^{5}+O\left(u^{6}\right) .
$$

Here $r$ is the bifurcation parameter and the transition from subcriticality to supercriticality occurs at $r=0$ when $27 f_{3}+38 f_{2}^{2}=0$.

To focus on the vicinity of this transition we write $r=\epsilon^{4} \mu$ and $f_{3}=-\frac{38}{27} f_{2}^{2}+\epsilon^{2} b$, where $\mu$ and $b$ are $O(1)$ unfolding parameters, and consider the following multiscale asymptotic expansion

$$
u(x, t) \sim \sum_{n=1}^{\infty} \epsilon^{n} u_{n}\left(x, X \equiv \epsilon^{2} x, T \equiv \epsilon^{4} t\right) .
$$


Substitution of this expansion into Eq. (A.1) leads to the following sequence of linear problems

$$
\begin{aligned}
M u_{1}= & 0 \\
M u_{2}= & f_{2} u_{1}^{2}, \\
M u_{3}= & -4\left(u_{1, x x}+u_{1}\right)_{X x}-\frac{38}{27} f_{2}^{2} u_{1}^{3}+2 f_{2} u_{2} u_{1} \\
M u_{4}= & -4\left(u_{2, x x}+u_{2}\right)_{X x}-\frac{38}{9} f_{2}^{2} u_{1}^{2} u_{2}+2 f_{2} u_{1} u_{3}+f_{2} u_{2}^{2}+f_{4} u_{1}^{4}, \\
M u_{5}= & -4\left(u_{3}+u_{3, x x}\right)_{X x}+2 f_{2}\left(u_{1} u_{4}+u_{2} u_{3}\right)+b u_{1}^{3}-\frac{38}{9}\left(u_{2}^{2}+u_{1} u_{3}\right) f_{2}^{2} u_{1} \\
& +\mu u_{1}-u_{1, T}-2 u_{1, X X}-6 u_{1, X X x x}+f_{5} u_{1}^{5}+4 f_{4} u_{1}^{3} u_{2} .
\end{aligned}
$$

Here $M$ is the linear self-adjoint operator $M \equiv\left(1+\partial_{x x}\right)^{2}$.

At each order the homogeneous problem $M u=0$ has four independent solutions, two that are periodic in $x$ and two that diverge as $|x| \rightarrow \infty$. Thus at each order the homogeneous solutions must take the form $A_{n}(X, T) e^{i x}+$ c.c., where the $A_{n}$ denote the corresponding amplitude. Without loss of generality we set $A_{1}=A(X, T)$ and $A_{n}=0, n>1$, and seek an evolution equation for $A(X, T)$.

The solutions $u_{n}$ for $n=1, \ldots, 4$ are

$$
\begin{aligned}
u_{1}= & A e^{i x}+\text { c.c. } \\
u_{2}= & f_{2}\left\{\frac{A^{2}}{9} e^{2 i x}+c . c .\right\}+2 f_{2}|A|^{2}, \\
u_{3}= & -\frac{f_{2}^{2} A^{3}}{54} e^{3 i x}+c . c . \\
u_{4}= & \left\{\left(f_{4}-\frac{40 f_{2}^{3}}{81}\right) \frac{A^{4} e^{4 i x}}{225}+\left(4 f_{4}-\frac{727 f_{2}^{3}}{81}\right) \frac{|A|^{2} A^{2} e^{2 i x}}{9}+\frac{16 i f_{2} A A_{X} e^{2 i x}}{27}+\text { c.c. }\right\} \\
& +\left(6 f_{4}-\frac{1118 f_{2}^{3}}{81}\right)|A|^{4} .
\end{aligned}
$$

At $O\left(\epsilon^{5}\right)$ the solvability condition yields the amplitude equation

$$
A_{T}=\mu A+4 A_{X X}+\frac{32 i f_{2}^{2}}{27}|A|^{2} A_{X}+3 b|A|^{2} A-2\left(\frac{1960 f_{2}^{4}}{81}-\frac{58 f_{2} f_{4}}{3}-5 f_{5}\right)|A|^{4} A .
$$

This equation can be identified with Eq. (1.1) after a rescaling, yielding

$$
a_{1}=\frac{4 f_{2}^{2}}{21 \sqrt{5}}\left(f_{2}^{4}-\frac{783}{980} f_{2} f_{4}-\frac{81}{392} f_{5}\right)^{-1 / 2}, \quad a_{2}=0
$$

provided $f_{2}^{4}-\frac{783}{980} f_{2} f_{4}-\frac{81}{392} f_{5}>0$.

\section{B. Derivation of the nonlinear phase equation}

Here we present a derivation of the nonlinear phase equation described in section 3.1. Before presenting the calculations, we remark that at the onset of phase instability $f=0$ is equivalent to $2 g_{0}=-b_{+} b_{-}$, where $b_{ \pm}$are given in Eq. (3.17). This relation will be used several times in the calculation to simplify expressions. Applying the scaling assumption introduced in Eq. (3.15) the $O\left(\epsilon^{2}\right)$ equation yields

$$
2 g_{0} r^{(0)}=b_{-} \phi_{X}^{(0)}
$$


indicating that the perturbed amplitude is slaved to the gradient of the phase. The $O\left(\epsilon^{3}\right)$ equation is automatically satisfied. At $O\left(\epsilon^{4}\right)$ and $O\left(\epsilon^{5}\right)$ one obtains, after using the relation in Eq. (B.1),

$$
\begin{aligned}
-2 g_{0} r^{(1)}+b_{-} \phi_{X}^{(1)}= & \partial_{X}^{3} \phi^{(0)} / b_{+}+\phi_{X}^{(0) 2}\left[\left(8 k_{0}+b_{-}\right) /\left(2 b_{+}\right)+\left(2 R_{0,+}^{2} b^{\prime}\right) / b_{+}^{2}+1\right] \\
& +\mu_{2} \phi_{X}^{(0)}\left[\left(a_{1}-a_{2}\right) \partial_{\mu} R_{0,+}^{2}-2\left(b^{\prime} \partial_{\mu} R_{0,+}^{2}+2\right) / b_{+}\right], \\
b_{+} r_{X}^{(1)}+\phi_{X X}^{(1)}= & \phi_{T}^{(0)}+\partial_{\mu} R_{0,+}^{2} \mu_{2}\left(a_{1}+a_{2}\right) \phi_{X X}^{(0)} / b_{+}+\left(b_{+}+4 k_{0}\right) \phi_{X X}^{(0)} \phi_{X}^{(0)} / b_{+}^{2} .
\end{aligned}
$$

Since $2 g_{0}=-b_{+} b_{-}$we see that Eq. (B.3) is proportional to the spatial derivative of Eq. (B.2). The nonlinear phase equation (3.16) now follows on eliminating $r^{(1)}$ between these two equations.

Acknowledgements. This work was supported in part by the National Science Foundation under grant DMS1211953.

\section{References}

[1] H.-C. Kao, E. Knobloch. Weakly subcritical stationary patterns: Eckhaus stability and homoclinic snaking, Phys. Rev. E 85 (2012), 026207.

[2] W. Eckhaus, G. Iooss. Strong selection or rejection of spatially periodic patterns in degenerate bifurcations, Physica D 39 (1989), pp. 124-146.

[3] A. Shepeleva. On the validity of the degenerate Ginzburg-Landau equation, Math. Method Appl. Sci. 20 (1997), pp. 1239-1256.

[4] H. Brand, R. Deissler. Eckhaus and Benjamin-Feir instabilities near a weakly inverted bifurcation, Phys. Rev. A 45 (1992), pp. 3732-3736.

[5] J. Burke, E. Knobloch. Localized states in the generalized Swift-Hohenberg equation, Phys. Rev. E 73 (2006), 056211.

[6] O. Batiste, E. Knobloch, A. Alonso, I. Mercader. Spatially localized binary-fluid convection, J. Fluid Mech. 560 (2006), pp. $149-158$.

[7] C. Beaume, A. Bergeon, E. Knobloch. Homoclinic snaking of localized states in doubly diffusive convection, Phys. Fluids 23 (2011), 094102.

[8] A. Doelman and W. Eckhaus. Periodic and quasi-periodic solutions of degenerate modulation equations, Physica D 53 (1991), pp. 249-266.

[9] J. Duan, P. Holmes. Fronts, domain walls and pulses in a generalized Ginzburg-Landau equation, Proc. Edinburgh Math. Soc. 38 (1995), pp. 77-97.

[10] A. Shepeleva. Modulated modulations approach to the loss of stability of periodic solutions for the degenerate GinzburgLandau equation, Nonlinearity 11 (1998), pp. 409-429.

[11] D. Henry. Geometric theory of semilinear parabolic equations, Springer-Verlag, Berlin, 1981.

[12] R. Hoyle. Pattern Formation, Cambridge University Press, Cambridge, 2006.

[13] J. Duan, P. Holmes, E. S. Titi. Global existence theory for a generalized Ginzburg-Landau equation, Nonlinearity 5 (1992), pp. 1303-1314.

[14] S. M. Cox, P. C. Matthews. Exponential time differencing for stiff systems, J. Comp. Phys. 176 (2002), pp. $430-455$.

[15] L. Kramer, W. Zimmermann. On the Eckhaus instability for spatially periodic patterns, Physica D 16 (1985), pp. 221232.

[16] E. Ben-Jacob, H. Brand, G. Dee, L. Kramer, J. S. Langer. Pattern propagation in nonlinear dissipative systems, Physica D 14 (1985), pp. 348-364.

[17] W. van Saarloos. Front propagation into unstable states. II. Linear versus nonlinear marginal stability and rate of convergence, Phys. Rev. A 39 (1989), pp. 6367-6390.

[18] J. Burke, J. H. P. Dawes. Localised states in an extended Swift-Hohenberg equation, SIAM J. Appl. Dyn. Syst. 11 (2012), pp. 261-284.

[19] L. J. Boya. Supersymmetric quantum mechanics: two simple examples, Eur. J. Phys. 9 (1988), pp. $139-144$.

[20] C. M. Elliott, S. Zheng. On the Cahn-Hilliard equation, Arch. Rational Mech. Anal. 96 (1986), pp. $339-357$.

[21] C. Beaume, A. Bergeon, H.-C. Kao, E. Knobloch. Convectons in a rotating fluid layer, J. Fluid Mech. 717 (2013), pp. $417-448$.

[22] E. Knobloch, J. DeLuca. Amplitude equations for travelling wave convection, Nonlinearity 3 (1990), pp. 975-980. 